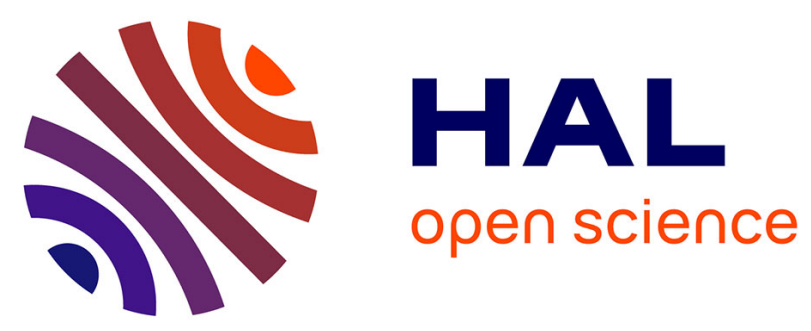

\title{
Antiviral activity of PLK1-targeting siRNA delivered by lipid nanoparticles in HBV-infected hepatocytes
}

Adrien Foca, Ammen Dhillon, Thomas Lahlali, Julie Lucifora, Anna Salvetti, Michel Rivoire, Amy Lee, David Durantel

\section{- To cite this version:}

Adrien Foca, Ammen Dhillon, Thomas Lahlali, Julie Lucifora, Anna Salvetti, et al.. Antiviral activity of PLK1-targeting siRNA delivered by lipid nanoparticles in HBV-infected hepatocytes. Antiviral Therapy, 2020, 25 (3), pp.151-162. 10.3851/IMP3361 . hal-03315938

\author{
HAL Id: hal-03315938 \\ https://hal.science/hal-03315938
}

Submitted on 12 Aug 2021

HAL is a multi-disciplinary open access archive for the deposit and dissemination of scientific research documents, whether they are published or not. The documents may come from teaching and research institutions in France or abroad, or from public or private research centers.
L'archive ouverte pluridisciplinaire HAL, est destinée au dépôt et à la diffusion de documents scientifiques de niveau recherche, publiés ou non, émanant des établissements d'enseignement et de recherche français ou étrangers, des laboratoires publics ou privés. 


\section{Antiviral activity of PLK1-targeting siRNA delivered by lipid nanoparticles in HBV-infected hepatocytes}

\section{Adrien Foca ${ }^{1,2}$, Ammen Dhillon ${ }^{3}$, Thomas Lahlali ${ }^{1,2}$, Julie Lucifora ${ }^{1,2}$, Anna Salvetti ${ }^{1,2}$, Michel Rivoire ${ }^{4}$, Amy Lee ${ }^{3}$, and David Durantel ${ }^{1,2,5, *}$}

1. Cancer Research Center of Lyon (CRCL), INSERM U1052, 69008 Lyon, France;

2. University of Lyon, Université Claude-Bernard (UCBL), UMR_S1052, UCBL, 69008 Lyon, France;

3. Arbutus Biopharma Corporation, 100-8900 Glenlyon Parkway, Burnaby, BC, Canada;

4. INSERM U1032, Centre Léon Bérard (CLB), Lyon, France

5. Labex DEVweCAN, 69008 Lyon, France.

* Corresponding author

\section{Correspondence:}

David Durantel, PhD, HDR

CRCL - INSERM U1052, 151 cours Albert Thomas, 69424 Lyon Cedex 03, France

Email: david.durantel@inserm.fr

Phone: + 33472681 970/+ 33472681959

Fax: +33472681971

\section{Manuscript information:}

Abstract: 247 words

Electronic word count: 3897

Number of table: 0

Number of figures: 5

Number of supplementary figures: 4

\section{Additional Footnotes:}

Involvement of authors:

- study concept and design: $A F, A D, A L$ and $D D$

- acquisition of data: $A F, A D, T L, J L$ and $D D$

- analysis and interpretation of data: $A F, A D, A L, J L$ and $D D$

- providing material: MR

- drafting of the manuscript: AF and DD

- critical revision of the manuscript for important intellectual content: $A F, A L, J L, A S$ and $D D$

- statistical analysis: AF and DD 


\section{Conflict of interest:}

DD received a research grant from Arbutus Biopharma for this work;

$A D$ and $A L$ are Arbutus Biopharma's employees;

$A S, J L$ and MR: no conflict of interest

\section{List of abbreviations (by alphabetical order):}

ApoB, apolipoprotein B; ATP, adenosine triphosphate; cccDNA, covalently-closed-circular DNA; CDNA, complementary DNA; CPAM, core protein allosteric modulator; $\mathrm{CHB}$, chronic hepatitis $\mathrm{B}$; d.p.i., days post infection; DAA, direct acting agent; dHepaRG, differentiated HepaRG cells; DMSO, dimethylsufoxide; EDTA, ethylenediaminetetraacetic acid; ELISA, enzyme-linked immunosorbent assay; FRG mice, $\mathrm{Fah}^{-/-} / \mathrm{Rag}^{-/-} / 112 \mathrm{rg}^{-/-}$mice; HBc, HBV core antigen; HBeAg, HBV e antigen; $\mathrm{HBsAg}$, HBV s antigen; HBV, hepatitis B virus; HCC, hepatocellular carcinoma; HTA, host targeting agent; IFN-a, interferon-alpha; IU, international unit; LHBS, large HBV $s$ antigen; LNP, lipid nanoparticles; MoA, mode of action; MOI, multiplicity of infection; mRNA, messenger RNA; NUCs, nucleos(t)ide analogs; PHH primary human hepatocytes; PLK1, polo-like-kinase1; pgRNA, pregenomic RNA; qPCR, quantitative polymerase chain reaction; rCDNA, relaxed circular DNA; RTqPCR, reverse transcriptase coupled quantitavite PCR; siRNA, small interfering ribonucleic acid; SM-PLK1i, small molecule PLK1 inhibitor; snRNA, small nuclear RNA; SoC, standard of care; tRNA, transfer RNA; TFV, tenofovir; vge, virus genome equivalent; vRNA, viral RNA; WHO, world health organization. 


\section{ABSTRACT}

A link between HBV and PLK1 was clearly evidenced in HBV-driven carcinogenesis, and we have also recently shown that PLK1 is a proviral factor in the early phases of HBV infection. Moreover, we have shown that $\mathrm{BI}-2536$, a small molecule PLK1 inhibitor, was very efficient at inhibiting HBV DNA neosynthesis, notably by affecting nucleocapsid assembly as a result of the modulation of $\mathrm{HBC}$ phosphorylation. Yet, as small molecule kinase inhibitors often feature poor selectivity, a more specific and safer strategy to target PLK1 would be needed for a potential development against chronic HBV infections. Here, we analysed using both freshly isolated primary human hepatocytes and differentiated HepaRG, the anti-HBV properties of an LNP-encapsulated PLK1targeting siRNA. Standard assays were used to monitor the effect of LNP siPLK1, or controls (LNP siHBV and LNP siNon-Targeting), on HBV replication and cell viability. A dose as low as $100 \mathrm{ng} / \mathrm{mL}$ of LNP-siPLK1 resulted in a $>75 \%$ decrease in secreted HBV DNA (viral particles), which was comparable to that obtained with LNP siHBV or $10 \mu \mathrm{M}$ of Tenofovir (TFV), without affecting cell viability. Interestingly, and in contrast to that obtained with TFV, a strong inhibition of viral RNA and $\mathrm{HBe} / \mathrm{HBsAg}$ secretions was also observed under LNP siPLK1 treatment. This correlated with a significant intracellular decrease of vRNA accumulation, which was independent of any change in cccDNA levels, thus suggesting a transcriptional or post-transcriptional modulation. Such an effect was not obtained with a biochemical approach of PLK1 inhibition, suggesting an enzymaticindependent role of PLK1.

\section{KEYWORDS}

Hepatitis B virus; Polo-like-kinase 1; small-interfering RNA, lipid nanoparticle; antiviral; hosttargeting agent; hepatocellular carcinoma. 


\section{INTRODUCTION}

Hepatitis B virus (HBV) infection represents the main cause of hepatocellular carcinoma (HCC), which is the $2^{\text {nd }}$ leading cause of cancer deaths worldwide (WHO data). Out of the hundred million people exposed to HBV, 257 million remain currently chronically infected (CHB) (WHO data; global hepatitis report, 2017). If left untreated, these patients have a 100 fold increased risk of developing HCC compared to the general population [1]. When patients are successfully and continiously treated with safe nucleos(t)ide analogs (NUCs), their risk of developing HCC is 2 to 5 times higher compared to normal individual[2,3]. This reminiscent risk of HCC under treatment is likely due to the fact that NUCs do not allow the elimination of the HBV replicative intermediate called cccDNA (i.e., covalently closed circular DNA), which is responsible for viral persistence and rebound when treatment is stopped $[4,5]$. In addition to the development of drugs that may target directly cccDNA $[4,5]$, the discovery of host-targeting agents (HTA) acting both on HBV replication and HCC initiation and/or progression could increase the efficacy of current standards of care $(\mathrm{SoC})$ for curing patients from the infection and associated liver diseases.

We previously identified the serine/threonine polo-like-kinase 1 (PLK1) as such a host target [6]. PLK1 is known to be overexpressed in many human cancers [7], and was demonstrated an important driver of HBV-induced HCC [8-11]. Moreover, we found that PLK1 is a proviral factor of HBV and that small inhibitory molecules of PLK1 (here after abbreviated SM-PLK1i) (e.g., BI-2536) had a negative impact on HBV replication [6]. The inhibition of PLK1 could therefore be of interest for the treatment of CHB patients with potential effects on both virologic and oncologic aspects of the disease.

Due to their poor specificity (i.e., they target several kinases, not a single one) and overall low safety profile, as demonstrated in the oncology field, SM-PLK1i cannot be reasonably clinically trialed in CHB patients, who require long-term and very safe treatments. Interestingly, small 
interfering RNAs directed against PLK1 were also shown to be efficient at targeting PLK1 oncogenic activity [12]. Chemically modified and injectable versions of PLK1 siRNA were also validated in preclinical cancer animal models $[13,14]$, and one PLK1 siRNA encapsulated in proprietary lipid nanoparticles (LNPS) was clinically trialed in patients with advanced HCC (NCT02191878).

In this study, we found that the use of LNP siPLK1 in HBV-infected primary human hepatocytes (PHHs) lead to a decrease in the amount of intracellular HBV RNAs, that subsequently affect the amount of secreted viral particles and $\mathrm{HBe} / \mathrm{HBs}$ antigens. Knowing that a loss of HBsAg in patients represents a clinical end-point goal [3], our finding could have relevant therapeutic implications. Moreover, these data point-out a novel PLK1 proviral function, which seems independent of its kinase activity. Indeed besides being involved in HBV reverse transcription through the regulation of capsid assembly [6], our data suggest that PLK1 could also be involved in the regulation of HBV RNA accumulation. 


\section{METHODS}

\section{Chemicals, antibodies, and others reagents}

PLK1 inhibitor BI-2536 was purchased from Selleckchem. All other chemicals were purchased from Sigma-Aldrich. Rabbit polyclonal anti-HBc antibodies were purchased from Dako (B0586) or Abcam (ab115992) and monoclonal anti-HBc antibodies from Abcam (ab8637; Clone C1). Rabbit polyclonal anti-PLK1 antibodies from Abcam (ab21738) were used for detection of phospho-PLK1$\mathrm{S}_{137}$; rabbit monoclonal anti-PLK1 antibodies from Abcam (ab115095) were used for detection of phospho-PLK1-T 210 and rabbit polyclonal anti-PLK1 antibodies from Abcam (ab109777) for detection of total PLK1.

\section{HepaRG and primary human hepatocyte cultures}

Human liver progenitor HepaRG cells $[15,16]$ were cultured and differentiated (dHepaRG) as previously described [17]. Primary human hepatocytes $(\mathrm{PHH})$ were freshly prepared from human liver resection obtained from the Centre Léon Bérard (Lyon) with French ministerial authorizations (AC 2013-1871, DC 2013 - 1870, AFNOR NF 96900 sept 2011) as previously described [18].

\section{Production of siRNA-containing liponanoparticles and use in cell culture}

The forward and reverse sequences of siRNA against PLK1 are respectively, 5'-

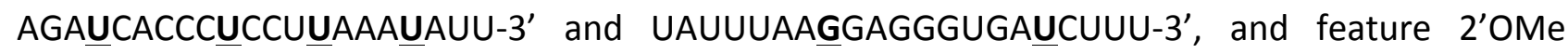
modification at indicated position (bold underlined) as previously described [14]. This dupleix siRNA was formulated in liponanoparticles as previously descrived [19]. The lipid formulation contains 1,2-dipalmitoyl-sn-glycero-3-phosphocholine,1,2-dilinoleyloxy-N,N-dimethylpropylamine, 3-N-[( $\omega$-methoxypoly(ethyleneglycol $\left.{ }_{2000}\right)$ carbamoyl]-1,2-dimyristyloxy-propylamine and cholesterol. 
The equivalence between molarity of siPLK1 and amount of LNP siPLK1 in $\mathrm{ng} / \mathrm{mL}$ is: $1 \mathrm{nM}$ of siPLK1 is equivalent to $13.3 \mathrm{ng} / \mathrm{mL}$ of LNP siPLK1. Controls LNP-siRNAs were manufactured the same way. The sequences of siRNA against HBV are Fw: 5'-CGACCUUGAGGCAUACUUCUU-3' and Rv: 5'GAAGUAGCCUCAAGGUCGUU-3' ON-TARGETplus duplex. LNP siRNAs were administrated to cells by direct dilution into the culture medium. Cells were exposed to LNPs for 24 hours, then washed with culture medium to remove excess of LNPs. Treatment were repeated as indicated in figure legends.

\section{HBV infection and analysis of viral replication}

Differentiated HepaRG cells (dHepaRG) and PHHs were infected with HBV genotype D (concentrated from HepAD38 cells supernatants) as described previously [20]. After treatment procedures, levels of intracellular and/or extracellular HBV RNAs and DNAs, HBc, secretion of $\mathrm{HBeAg}$ and $\mathrm{HBsAg}$ were monitored by quantitative polymerase chain reaction (qPCR), reverse transcription qPCR (RT-qPCR), immunoblot and enzyme-linked immunosorbent assay (ELISA) respectively, as previously described $[6,20]$. Briefly, $\mathrm{HBeAg}$ and $\mathrm{HBsAg}$ were quantified in culture medium using a chemiluminescence immunoassay kit (Autobio Diagnostics) according to manufacturer's instructions. Total intracellular DNAs and RNAs as well as secreted viral DNAs/RNAs were purified from infected cells using NucleoSpin ${ }^{\circledR} 96$ Tissue kit, NucleoSpin ${ }^{\circledR} 96$ RNA kit, NucleoSpin ${ }^{\circledR} 96$ virus kit (Macherey-Nagel) respectively, according to manufacturer's instructions. Reverse-transcription of RNA into cDNA was performed with the SuperScript ${ }^{\circledR}$ III First-Strand Synthesis kit (Invitrogen) according to manufacturer's instructions. All qPCR analyses were performed with LightCycler ${ }^{\mathrm{TM}} 96$ and 480 systems (Roche), except for cccDNA and pgRNA specific PCRs that were processed under a QuantStudio ${ }^{\mathrm{TM}} 7$ (Applied), using homemade TaqMan assays [20]. 
This study was approved by the WuXi IACUC (Institutional Animal Care and Use Committee). FRG ${ }^{\text {TM }}$ $\left(\mathrm{Fah}^{-/-} / \mathrm{Rag}^{-/-} / \mathrm{Il} 2 \mathrm{rg}^{-/-}\right)$mice were purchased from Yecuris (Tualatin, OR, USA), engraphted with PHH and infected with $1.10^{9}$ virus genome equivalent (vge) of HBV genotype D. When HBV viremia had reached a maximum (i.e., around $10 \mathrm{e} 9 \mathrm{vge} / \mathrm{mL}$ ), the liver was digested by in situ collagenase perfusion and recovered hepatocytes seeded in DMEM medium into 24-well plates $\left(2.7 .10^{5}\right.$ cells/well). LNP treatments were conducted every 4 days starting at day 0 and $\mathrm{BI}-2536$ treatment was started on day 0 and replenished every tow days. DNA in the culture supernatants was isolated using QIAamp 96 DNA Blood Kit (Qiagen) and quantified by qPCR. HBsAg was quantified using specific CLIA kit (Autobio).

\section{Separation of cytoplasm and nucleopasm}

Extractions of nuclear and cytosolic proteins were performed using NE-PER Nuclear and Cytoplasmic kit (Thermo Scientific), according to the manufacturer's instructions. Sodium orthovanadate, Complete ${ }^{\mathrm{TM}}$ EDTA-free protease inhibitor cocktail (Roche) and sodium fluoride were added freshly to all lysates. Nuclear and cytosolic RNAs were isolated as described in Weil et al., [21], followed by a purification with TRI reagent (Merck) and precipitation with isopropanol. Purity of nuclear vs cytoplasmic extracts, was assessed by RT-qPCR using primers to amplify the

nuclear U6 snRNA (Fw: 5'-CTCGCTTCGGCAGCACATATAC-3' / Rv: 5'GGAACGCTTCACGAATTTGCGTG-3') and the cytoplasmic Human Tyr-tRNA (Fw: 5'-

cCtTCGATAGCTCAGCTGGTAGAGCGGAGG-3' / Rv: 5'-CGgAATTGAACCAGCGACCTAAGGATGTCC$\left.3^{\prime}\right)$.

\section{Capsid migration assay}

The intracellular HBV nucleocapsids were analyzed by agarose electrophoresis in native condition followed by transfer onto an enhanced chemiluminescence membrane (Amersham) and western blot analysis, as previously described [20]. 


\section{Southern Blotting}

Total DNAs extracted with NucleoSpin ${ }^{\circledR} 96$ Tissue kit (Macherey-Nagel) were pooled, precipitated using $5 \mathrm{M} \mathrm{NaAc}$ and ethanol and analyzed by Southern blot as previously described [22]. DNAs from HepG2-NTCP cells extracted using a modified Hirt procedure were used as positive controls [23].

\section{Statistical analysis}

Statistical analysis was performed using two-way analysis of variance, $t$ tests, or non-parametric Mann-Whitney tests using GraphPad Prism software, version 7.04. For all tests, $\mathrm{P} \leq 0.05^{*}, \mathrm{P} \leq$ $0.01^{* *}$, and $\mathrm{P} \leq 0.001^{* * *}$ were considered statistically significant. 


\section{RESULTS}

\section{Efficacy and safety of LNP siPLK1 in hepatocytes}

Compared to control LNP siRNAs, LNP siPLK1 were efficient at inhibiting PLK1 expression, with a $70 \%$ loss of PLK1-mRNA at $13.3 \mathrm{ng} / \mathrm{mL}$ without toxicty in proliferating HepG2 cells. As expected, LNP siPLK1 induced a $50 \%$ mortality in proliferating HepG2 cells at $133 \mathrm{ng} / \mathrm{mL}$ due to active cell division and crucial role of PLK1 in G2/M transition (Fig. Sup. 1). Considering these data, as well as the concentration correspondence (i.e., $1 \mathrm{nM}$ of naked siPLK1 is equivalent to $13.3 \mathrm{ng} / \mathrm{mL}$ of LNP siPLK1) and our previous work done using naked siRNA targeting PLK1 [6], we tested the effect of LNP siPLK1 and controls in HBV-infected primary human hepatocytes, and related dHepaRG cells, with a range of concentration going from 100 to $300 \mathrm{ng} / \mathrm{mL}$. We observed a strong reduction in the levels of PLK1 mRNA in both dHepaRG and PHH (Fig. Sup. 2A and Fig. 1A) and in the level of PLK1 phosphoprotein in PHH (Fig. 1B). Interestingly, as previously reported, LNP siHBV led also to a reduction of PLK1 mRNA and PLK1 phosphorylation to levels comparable to those observed in uninfected cells (compare lanes LNP-siHBV and NI; Fig. 1A and 1B), thus confirming that HBV strongly increases PLK1 activity in PHH. Confirming also our previously published data [6], the inhibition of PLK1 activity was not cytotoxic in PHH (3 different batches tested) even when LNP siPLK1 was administrated 3 times at 300ng/mL (Fig. 1C). Moreover PHH remained functional, as measured by the apolipoprotein $B(A p o B)$ secretion (see apoB dosing in Fig. 2A), a component of VLDL (very low density lipoprotein) that are made by healthy hepatocytes.

LNP siPLK1 strongly, and specifically, inhibits viral particle and antigen secretion in HBV-infected primary human hepatocytes

To evaluate the antiviral activity of LNP siPLK1, freshly isolated PHH from three different donors were infected with HBV at a high multiplicity of infection, and at day-4 post-infection (p.i.), when the average $\mathrm{HBsAg}$ production reached around $1000 \mathrm{IU} / \mathrm{mL}$ (which is a level signing a high 
replication of HBV), cells were treated three times every three days with increasing LNP siPLK1 doses or control molecules. Secretion of viral DNA (i.e., infectious virion) and RNA (i.e., RNAcontaining particles; [24]) were significantly inhibited by LNP siPLK1 (Fig. 2A). These inhibitons were comparable to those observed with LNP siHBV in the model. The lack of dose-dependency of the phenotype suggests that even a weak loss of PLK1/phospho-PLK1 (Fig. 1B) was associated with a strong inhibition of virus replication. Interestingly, we also observed a $70 \%$ inhibition of viral antigen $\mathrm{HBeAg}$ and $\mathrm{HBsAg}$ secretion (Fig. 2A), without any effect on $\mathrm{ApoB}$ secretion, highlighting a specificity of LNP siPLK1 action on those secreted viral proteins. In the mean time, tenofovir (TFV) did not affect HBV antigens secretion and inhibition of HBV antigen secretion was stronger with LNP siHBV, as expected due to the direct targeting of HBV RNAs encoding those antigens.

This strong inhibition of $\mathrm{HBeAg}$ and $\mathrm{HBsAg}$ secretion was also confirmed in the HBV-infected dHepaRG cells with the same experimental setting (Fig. Sup. 2B). Moreover, the inhibitory phenotype on virion and HBsAg secretion was also observed by ex vivo treatment with LNP siPLK1 of freshly isolated hepatocytes from HBV-infected liver-humanized FRG mice [25], which replicate HBV at high level (i.e., around $1.10^{9}$ copies $/ \mathrm{mL}$ at day-26 post-infection), mimicking the highly viremic "immune tolerant » phase in human. In this model, $300 \mathrm{ng} / \mathrm{mL}$ of LNP siPLK1 led to a decrease of $60 \%$ in HBV DNA and $65 \%$ in $\mathrm{HBsAg}$, whereas treatment with LNP siHBV was very efficient (Fig. Sup. 3).

LNP SIPLK1 induces a reduction of intracellular viral RNA accumulation without significant modification of cccDNA amount

To further describe the anti-HBV potential of LNP siPLK1 and get insigths on the observed phenotype on secreted HBV antigens, intracellular viral parameters were also analyzed. We observed a dose dependent decrease of intracellular HBV DNAs and RNAs in HBV-infected PHH or dHepaRG cells treated with LNP siPLK1 (Fig. 2B and Fig. Sup. 2C). The highest dose of LNP siPLK1 
resulted in a decrease of 55\% (70\% in dHepaRG) and 50\% (also 50\% in dHepaRG) of HBV DNA and RNA respectively (Fig. 2B and Fig. Sup. 2C). LNP siHBV treatment resulted in a stronger effect, with $80 \%$ and $90 \%$ drops, whereas only viral DNA amount decreased with TFV (65\% in $\mathrm{PHH}$ and $90 \%$ in dHepaRG) as expected. The lower inhibition of intracellular DNA amount for all molecules tested, as compared to what observed with secreted DNA is likely due to the detection of remaining input HBV DNA from HBV inoculum as discussed below. Interestingly and contrary to what we described with SM-PLK1i [6], we observed a decrease in the levels of HBV RNAs upon LNP siPLK1 treatment of HBV-infected hepatocytes without observing any significant changes in cccDNA amount (analyzed by qPCR or Southern Blot) (Fig. 3). These data suggest that the decrease of HBV RNA observed with LNP siPLK1 is a consequence of a transcriptional and/or post-trancriptional event.

\section{LNP siPLK1 does not inhibit better HBV replication in pre-infection treatment setting}

To determine if PLK1 affects HBV infection establishment, we treated PHH twice with LNP siPLK1 or control molecules, prior to HBV infection and a third treatment was done at the time of HBV inoculation in order to maintain PLK1 protein level low from HBV entry to ccCDNA formation. Analyses were done at 5d.p.i. PLK1 mRNA knockdown was still $>75 \%$ with 100 and $300 \mathrm{ng} / \mathrm{mL}$ of LNP siPLK1 (Fig. 4A). As seen before LNP siHBV allowed a PLK1 mRNA decrease, in accordance to the level found in uninfected cells. Interestingly a similar phenotype was observed with an entry inhibitor (i.e., PreS1 peptide), which very efficiently prevented HBV infection in this setting (as shown in other panels of Fig. 4). Regarding antiviral phenotypes obtained with LNP siPLK1, they were very similar to that obtained in post-infection treatment setting (compare results of Fig. $\mathbf{2}$ and 4). Importantly, cccDNA establishment was not fully blocked by either LNP siPLK1 or LNP siHBV, as compared to that observed with PreS1 peptide, which acts a genuine entry inhibitor. The slight decrease amount of cccDNA observed with LNP siPLK1 and LNP siHBV was poorly significant compared to controls (LNP si Ctrl and TFV), and could therefore not explain per se other observed 
inhibitory phenotypes. It is worth mentioning here that PreS1 peptide did not lead to an absence of detection of intracellular HBV DNA in this setting, as compared to other papameters (cccDNA, intracellular RNA, HBeAg, HBsAg), thus confirming that residual HBV DNA from inoculation perturbs analyses, as mentioned earlier. This is the reason why results obtained from supernatant analyses are more reliable, and without ambiguity demonstrate the overall strong effect of LNP siPLK1 on HBV replication.

\section{PLK1 knockdown results in a partial nuclear vRNA sequestration in HBV-infected PHH}

In HBV-infected PHH, activated pPLK1-S ${ }_{137}$ was mainly localized in the nucleus (Fig. Sup. 4 and refer also to [6]). As we observed a decrease in HBV RNAs in absence of change in the level of cCCDNA under LNP siPLK1 treatment, we wondered if PLK1 could be involved in the modulation of HBV RNA levels. To address this, we infected PHHs, treated them as above and harvested cells three days after the last treatment. We performed a cytosolic/nuclear fractionation in order to quantify HBV RNAs in both compartiments. RNA purity of our extracts was checked using nuclearspecific U6 snRNA and cytosolic-specific Tyr-tRNA. LNP siHBV resulted in a decrease of HBV RNAs levels in both compartments, whereas LNP siPLK1 led to a dual phenotype, depending on subcellular compartments. Indeed, in the cytosol, a 50\% decrease of HBV RNAs levels was observed, similar to that observed with whole cell extracts (Fig. 5). By contrast, in the nuclear compartment, a dose-dependent accumulation of HBV RNAs was observed under LNP siPLK1 treatment (Fig. 5C). As the amount of cytosolic HBV RNAs was overall 10 times higher than that observed in the nucleus, this accumulation of HBV RNAs in the nucleus induced by LNP siPLK1 did not modify the overall observed phenotype (i.e., reduction of total RNA accumulation), but certainly accounted for the decrease in viral protein synthesis. 


\section{DISCUSSION}

Hepatocellular carcinoma ranks second in term of cancer-induced death worldwide, with an estimated 600,000 death/year burden. HCC development is the consequence of the interplay between genetic predispositions, environmental factors (e.g., exposure to aflatoxins, alcohol, etc...), as well as oncogenic viruses; the latter are involved in $80 \%$ of cases, and HBV for itself accounts for $50 \%$ of HCC worlwide. As 257 million people are chronically infected with HBV and only a minority of them $(<1 \%)$ are effectively diagnosed and treated, the HBV-mediated HCC burden is far from being eradicated. If a better diagnostic of infected patients and a more universal use of antiviral therapies are needed, it would be also intresting to identify novel molecules that could target both viral replication and hepatocellular cancer cell growth.

Our previous study demonstrated that PLK1 is activated upon HBV infection and acts as a proviral factor. Consequently, we showed that its inhibition by BI-2536, a SM-PLK1i, leads to an inhibition of HBV replication. In addition, the group of $\mathrm{Dr} \mathrm{O}$. Andrisani have shown in several studies that PLK1, which is induced by $\mathrm{HBx}$, is a driver of murine hepatocyte cancerous transformation in vitro and that its inhibition by $\mathrm{BI}-2536$ could prevent it $[11,26]$. Moreover PLK1 being a validated target in oncology, important research efforts to identify potent SM-PLK1i were made, as reviewed recently [27]. However most of SM-PLK1i are not strictly specific to PLK1 itself and cross-inhibit of other kinase functions; this may account for the poor toxicologic profiles and side effects of these chemical inhibitors. The possibility to use siRNA to more specifically target kinases involved in cancer has opened novel perspectives. In this line, the clinical trial NCT02191878 performed with TKM-080301 in subjects with advanced HCC was instrumental and led us to consider LNP siPLK1 as a good candidate for early inhibition of HBV replication, in the dual perspective of use described above. 
Here we confirmed that PLK1 is crucial for HBV replication in non-dividing hepatocytes by using PLK1-targeting-siRNAs encapsulated in lipid nanoparticles (Arbutus Bioparma proprietary composition; the LNP-siPLK1 used in this study was closely related, but different from TKM080301). In particular, we demonstrated that LNP siPLK1 inhibits more efficiently HBV replication than naked siRNA or SM-PLK1i (reported in [6]) in the absence of cell toxicity. Indeed 100 to $300 \mathrm{ng} / \mathrm{mL}$ of LNP siPLK1 (corresponding to 7.5 to $22.5 \mathrm{nM}$ siPLK1) led to a $>75 \%$ decrease in HBV viremia in vitro. Moreover, in contrast to BI-2536, the SM-PLK1i that was previously profiled in depth in vitro and in liver-humanized FRG mice [6], we found that LNP siPLK1 was also capable to potently inhibit the secretion of HBV antigens in the supernatant of treated cells. This indirectly indicates that a kinase-independent function of PLK1 could account for production and secretion of HBV antigens. PLK1 could interact with a viral or host factor independently of its kinase activity and through this interaction contribute to an optimal production of HBV antigens. This proteinprotein interaction model to explain activitywas precedently exemplified for PLK1 by Vitour and colleagues [28]. In their study, it was shown that the polo-box domain of PLK1 could bind to the mitochondria-bound innate immunity adapter MAVS and inhibit its ability to activate the IRF3 and NF-kappaB pathways, without any requirement of the kinase activity.

Such an unexpected phetotype of LNP siPLK1 on HBV antigens production is particularly relevant, as the next step for the improvement of current HBV therapy in clinic is precisely to be capable to reduced antigenemia in patients, in particular $\mathrm{HBsAg}$, to potentially restore immune responses and control infection [3]. The LNP siPLK1-induced inhibition of HBeAg and HBsAg in supernatant correlated with a reduced accumulation of intracellular HBV RNAs in LNP siPLK1 treated cells, in the absence of any change in cccDNA amount. This observation points out toward a role of PLK1 in transcriptional and/or post-transcriptionnal regulation. In this study, we limited our work to the analysis of the impact of LNP siPLK1 on the accumulation of viral RNA in nucleoplasm and cytoplasm after fractionation, and found that HBV RNAs tended to accumulate in the nucleus 
under LNP siPLK1 treatment, while the total amount of RNA was decreased. Although the precise underlying mechanistic of this transcriptional and/or post-transcriptional regulation (i.e., cccDNA activity, vRNA stability, vRNA trafficking...) is yet to be defined, it is tempting to speculate that the previously described interplay between PLK1 and HBc [6] could also be at work. Indeed, HBV core protein is known to interact with cccDNA [29] and potentially in the regulation of transcription [30]. Moreover, it has been suggested that the HBc CTD could be determinant for HBc-cccDNA interaction [30] and in our precedent study, we have shown that PLK1 targets HBC CTD. Finally, $\mathrm{HBc}$ is also known to be an RNA-binding protein physically interacting with vRNAs. Of course, a role of PLK1 independently of HBc should also be considered. In this case PLK1 could interact with other known or unknown host-regulators of both/either cccDNA transcription and/or posttranscriptional events, and regulate their functions in a kinase-independent manner. Whether this is yet to be demonstrated regarding the pro-HBV role of PLK1, there are in literature many exemples of kinases, which feature kinase-independent regulatory functions [31-36]. Taken together, this provided a nice hypothetical landscape for further mechanistic studies to document LNP siPLK1 mode of action (MoA).

Of course, it would also be important to evaluate the anti-HBV efficacy of LNP SiPLK1 in a liver humanized mouse model to confirm its potential therapeutic interest on the virology side, and to compare it or combine it to siRNA targeting HBV itself. Whether siRNA targeting HBV are currently in clinical trials in CHB patients (e.g., phase-2 for ALN-HBV, NCT02826018; phase-2 for JNJ-3989 ,NCT03982186 and NCT04129554) and remains the most straightforward and relevant RNAi option against HBV replication, the co-administration of siRNAs targeting both HBV and PLK1 could be an original way to further improve such a therapeutic approach.

Regarding the possibility to test the relevance of using LNP siPLK1 as a double bullet compound, which would be able to both contribute to HBV replication inhibition in "add on therapy" with SoC 
and prevention of HBV-driven carcinogenesis, we would need a preclinical model able to recapitulate the entire pathogenesis process. A mouse model based on immune-competent mice transduced with AAV-HBV vectors could be of interest, as it features a persistent viral replication [37], in the context of an HBV immune tolerance [38], and was also reported to support HCC development [39]. Alternatively other transgenic mouse models featuring the overexpression of HBV proteins, HCC development and a demonstrated implication of PLK1 in carcinogenesis could be used to this end. Recently, it was shown that genomic integrity of hepatocytes was disrupted by viral LHBS and that PLK1 acted as a major modulator in LHBS-mediated G2/M checkpoint override. In this model, SM-PLK1i resulted in a G2/M checkpoint restauration and suppression of tumorigenesis [40].

To summarize, in this study we have confirmed the proHBV role of PLK1 and reported a potent and specific antiviral activity of LNP siPLK1 in HBV-infected hepatocytes, which includes a strong loss of intracellular vRNA accumulation, leading to reduction of HBV antigens secretion. As it is now rather well accepted that PLK1 is an important HCC driver, its inhibition by RNA interference in patients featuring an HCC from HBV etiology, in which HBV replication would be still active, could result in a combined effect on the virus and cancer cell growth. These results warrant additional investigations in preclinical animal model to further determine the usefulness of such a therapeutic strategy. 


\section{ACKNOWLEDGEMENTS}

The authors thank Maud Michelet, Océane Floriot, Jenifer Molle, Laura Dimier, Anaëlle Dubois and Judith Fresquet for PHHs isolation as well as the staff from Michel Rivoire's surgery room for providing us with fresh liver resections.

This work was supported by a grant from ANRS (French national agency for research on AIDS and viral hepatitis; CSS4) and by INSERM core grant to DD. This work was also supported by the DEVweCAN LABEX (ANR-10-LABX-0061) of the "Université de Lyon", within the program "Investissements d'Avenir" (ANR-11-IDEX-0007) operated by the French National Research Agency (ANR) to DD. The work was also supported by a research contract with Arbutus Biopharma.

\section{REFERENCES}

[1] Chan SL, Wong VWS, Qin S, Chan HLY. Infection and Cancer: The Case of Hepatitis B. Journal of Clinical Oncology 2015.

[2] Yoo J, Hann HW, Coben R, Conn M, DiMarino AJ. Update Treatment for HBV Infection and Persistent Risk for Hepatocellular Carcinoma: Prospect for an HBV Cure. Diseases 2018;6.

[3] Durantel D, Zoulim F. New antiviral targets for innovative treatment concepts for hepatitis B virus and hepatitis delta virus. Journal of hepatology 2016;64:S117-S131.

[4] Bloom K, Maepa MB, Ely A, Arbuthnot P. Gene Therapy for Chronic HBV-Can We Eliminate cccDNA? Genes 2018;9.

[5] Lucifora J, Protzer U. Attacking hepatitis B virus cccDNA--The holy grail to hepatitis B cure. Journal of hepatology 2016;64:S41-S48.

[6] Diab AM, Foca A, Fusil F, Lahlali T, Jalaguier P, Amirache F, et al. Polo-like-kinase 1 is a proviral hostfactor for hepatitis B virus replication. Hepatology 2017.

[7] Strebhardt K. Multifaceted polo-like kinases: drug targets and antitargets for cancer therapy. Nature reviews Drug discovery 2010;9:643-660.

[8] Studach LL, Menne S, Cairo S, Buendia MA, Hullinger RL, Lefrancois L, et al. Subset of Suz12/PRC2 target genes is activated during hepatitis $B$ virus replication and liver carcinogenesis associated with $\mathrm{HBV} X$ protein. Hepatology 2012;56:1240-1251.

[9] Wang WH, Studach LL, Andrisani OM. Proteins ZNF198 and SUZ12 are down-regulated in hepatitis B virus (HBV) $X$ protein-mediated hepatocyte transformation and in HBV replication. Hepatology 2011;53:1137-1147. 
[10] Andrisani OM, Studach L, Merle P. Gene signatures in hepatocellular carcinoma (HCC). Seminars in cancer biology 2011;21:4-9.

[11] Studach LL, Rakotomalala L, Wang WH, Hullinger RL, Cairo S, Buendia MA, et al. Polo-like kinase 1 inhibition suppresses hepatitis $B$ virus $X$ protein-induced transformation in an in vitro model of liver cancer progression. Hepatology 2009;50:414-423.

[12] Spankuch-Schmitt B, Bereiter-Hahn J, Kaufmann M, Strebhardt K. Effect of RNA silencing of pololike kinase-1 (PLK1) on apoptosis and spindle formation in human cancer cells. Journal of the National Cancer Institute 2002;94:1863-1877.

[13] Yao YD, Sun TM, Huang SY, Dou S, Lin L, Chen JN, et al. Targeted delivery of PLK1-siRNA by ScFv suppresses Her2+ breast cancer growth and metastasis. Science translational medicine 2012;4:130ra148.

[14] Judge AD, Robbins M, Tavakoli I, Levi J, Hu L, Fronda A, et al. Confirming the RNAi-mediated mechanism of action of siRNA-based cancer therapeutics in mice. The Journal of clinical investigation 2009;119:661-673.

[15] Marion MJ, Hantz O, Durantel D. The HepaRG cell line: biological properties and relevance as a tool for cell biology, drug metabolism, and virology studies. Methods in molecular biology 2010;640:261-272.

[16] Gripon P, Rumin S, Urban S, Le Seyec J, Glaise D, Cannie I, et al. Infection of a human hepatoma cell line by hepatitis B virus. Proc Natl Acad Sci U S A 2002;99:15655-15660.

[17] Hantz O, Parent R, Durantel D, Gripon P, Guguen-Guillouzo C, Zoulim F. Persistence of the hepatitis B virus covalently closed circular DNA in HepaRG human hepatocyte-like cells. J Gen Virol 2009;90:127-135.

[18] Lecluyse EL, Alexandre E. Isolation and culture of primary hepatocytes from resected human liver tissue. Methods in molecular biology 2010;640:57-82.

[19] Jeffs LB, Palmer LR, Ambegia EG, Giesbrecht C, Ewanick S, MacLachlan I. A scalable, extrusion-free method for efficient liposomal encapsulation of plasmid DNA. Pharmaceutical research 2005;22:362-372.

[20] Lahlali T, Berke JM, Vergauwen K, Foca A, Vandyck K, Pauwels F, et al. Novel potent capsid assembly modulators regulate multiple steps of the Hepatitis B virus life-cycle. Antimicrobial agents and chemotherapy 2018.

[21] Weil D, Boutain S, Audibert A, Dautry F. Mature mRNAs accumulated in the nucleus are neither the molecules in transit to the cytoplasm nor constitute a stockpile for gene expression. Rna 2000;6:962-975.

[22] Lucifora J, Salvetti A, Marniquet X, Mailly L, Testoni B, Fusil F, et al. Detection of the hepatitis B virus (HBV) covalently-closed-circular DNA (cccDNA) in mice transduced with a recombinant AAV-HBV vector. Antiviral research 2017;145:14-19.

[23] Cai D, Nie H, Yan R, Guo JT, Block TM, Guo H. A southern blot assay for detection of hepatitis B virus covalently closed circular DNA from cell cultures. Methods in molecular biology 2013;1030:151-161.

[24] Wang J, Shen T, Huang X, Kumar GR, Chen X, Zeng Z, et al. Serum hepatitis B virus RNA is encapsidated pregenome RNA that may be associated with persistence of viral infection and rebound. Journal of hepatology 2016;65:700-710.

[25] Bissig KD, Wieland SF, Tran P, Isogawa M, Le TT, Chisari FV, et al. Human liver chimeric mice provide a model for hepatitis $B$ and $C$ virus infection and treatment. The Journal of clinical investigation 2010;120:924-930.

[26] Studach L, Wang WH, Weber G, Tang J, Hullinger RL, Malbrue R, et al. Polo-like kinase 1 activated by the hepatitis $B$ virus $X$ protein attenuates both the DNA damage checkpoint and DNA repair resulting in partial polyploidy. The Journal of biological chemistry 2010;285:30282-30293.

[27] Liu Z, Sun Q, Wang X. PLK1, A Potential Target for Cancer Therapy. Translational oncology 2017;10:22-32.

[28] Vitour D, Dabo S, Ahmadi Pour M, Vilasco M, Vidalain PO, Jacob Y, et al. Polo-like kinase 1 (PLK1) regulates interferon (IFN) induction by MAVS. The Journal of biological chemistry 2009;284:21797-21809.

[29] Bock CT, Schwinn S, Locarnini S, Fyfe J, Manns MP, Trautwein C, et al. Structural organization of the hepatitis B virus minichromosome. Journal of molecular biology 2001;307:183-196.

[30] Chong CK, Cheng CYS, Tsoi SYJ, Huang FY, Liu F, Seto WK, et al. Role of hepatitis B core protein in HBV transcription and recruitment of histone acetyltransferases to cccDNA minichromosome. Antiviral research 2017;144:1-7.

[31] Audetat KA, Galbraith MD, Odell AT, Lee T, Pandey A, Espinosa JM, et al. A Kinase-Independent Role for Cyclin-Dependent Kinase 19 in p53 Response. Molecular and cellular biology 2017;37. 
[32] Jang KH, Jang T, Son E, Choi S, Kim E. Kinase-independent role of nuclear RIPK1 in regulating parthanatos through physical interaction with PARP1 upon oxidative stress. Biochimica et biophysica acta Molecular cell research 2018;1865:132-141.

[33] Li Q, Pene V, Krishnamurthy $\mathrm{S}$, Cha $\mathrm{H}$, Liang TJ. Hepatitis $\mathrm{C}$ virus infection activates an innate pathway involving IKK-alpha in lipogenesis and viral assembly. Nature medicine 2013;19:722-729.

[34] Sulzmaier FJ, Jean C, Schlaepfer DD. FAK in cancer: mechanistic findings and clinical applications. Nature reviews Cancer 2014;14:598-610.

[35] Vivanco I, Chen ZC, Tanos B, Oldrini B, Hsieh WY, Yannuzzi N, et al. A kinase-independent function of AKT promotes cancer cell survival. eLife 2014;3.

[36] Wang Z, Fu M, Wang L, Liu J, Li Y, Brakebusch C, et al. p21-activated kinase 1 (PAK1) can promote ERK activation in a kinase-independent manner. The Journal of biological chemistry 2013;288:20093-20099.

[37] Dion S, Bourgine M, Godon O, Levillayer F, Michel ML. Adeno-associated virus-mediated gene transfer leads to persistent hepatitis B virus replication in mice expressing HLA-A2 and HLA-DR1 molecules. Journal of virology 2013;87:5554-5563.

[38] Martin P, Dubois C, Jacquier E, Dion S, Mancini-Bourgine M, Godon O, et al. TG1050, an immunotherapeutic to treat chronic hepatitis $B$, induces robust $T$ cells and exerts an antiviral effect in HBVpersistent mice. Gut 2015;64:1961-1971.

[39] Huang YH, Fang CC, Tsuneyama K, Chou HY, Pan WY, Shih YM, et al. A murine model of hepatitis Bassociated hepatocellular carcinoma generated by adeno-associated virus-mediated gene delivery. International journal of oncology 2011;39:1511-1519.

[40] Li TN, Wu YJ, Tsai HW, Sun CP, Wu YH, Wu HL, et al. Intrahepatic hepatitis B virus large surface antigen induces hepatocyte hyperploidy via failure of cytokinesis. The Journal of pathology 2018;245:502513. 


\section{FIGURE LEGENDS}

Figure 1. Evaluation of the efficacy and toxicity of LNP siPLK1 in primary human hepatocytes. PHHs were left uninfected (non-infected control; NI) or were infected with HBV genotype D at a multiplicity of infection of 200 vge/cell. From 4 days post-infection (p.i.), cells were mock-treated (non treated control; NT) or treated 3 times 3 days apart with increasing LNP siPLK1 concentrations or the indicated control molecules (LNP siHBV and LNP siCtrl were used at $200 \mathrm{ng} / \mathrm{mL}$ and $10 \mu \mathrm{g} / \mathrm{mL}$ of puromycin served as positive death control, Tenofovir (TFV) was used at a concentration of $10 \mu \mathrm{M}$ ). Cells and supernatants were harvested at day-13 p.i. (day-9 posttreatment) for subsequent analyses. (A) RNAs were extracted and levels of PLK1 mRNAs (relative to PRNP mRNAs) analyzed by RT-qPCR. Results were normalized to the NT condition. (B) Proteins were extracted from cytosol and nuclear compartments with NE-PER Nuclear and Cytoplasmic kit. Thirty $\mu \mathrm{g}$ of nuclear fraction for each condition were analyzed by western blot. Lamin B1 served as loading control for nuclear proteins while $\beta$-Tubulin served as control for cytoplasm contamination (a representative blot is shown). (C) ATP production, reflecting cell viability, was measured using Cell TiterGlo One Solution Assay. Results presented in graphs are the mean +/- SEM (standard error of the mean) of 3 independent experiments ( 3 donors of $\mathrm{PHH}$ ) each performed in biological triplicate. $\mathrm{P} \leq 0.05^{*}, \mathrm{P} \leq 0.01^{* *}$, and $\mathrm{P} \leq 0.001^{* * *}$ were considered statistically significant.

Figure 2. Antiviral activity of LNP siPLK1 in non-transformed and non-dividing HBV-infected PHH. $\mathrm{PHH}$ were left uninfected (non-infected control; NI) or were infected with HBV genotype D at a multiplicity of infection of 200 vge/cell. From 4 days p.i., cells were mock-treated (non treated control; NT) or treated 3 times 3 days apart with indicated molecules. LNP siHBV and LNP siCtrl were used at $200 \mathrm{ng} / \mathrm{mL}$ and TFV at $10 \mu \mathrm{M}$. (A) Three days after the last treatment (day-13 postinfection/day-9 post-treatment), secreted viral parameters were measured in the supernatants. RNA and DNA were extracted from viral particles and analyzed by HBV-specific RT-qPCR or qPCR, 
whereas levels of secreted $\mathrm{ApoB}, \mathrm{HBeAg}$ and $\mathrm{HBsAg}$ were assessed by ELISA. (B) Intracellular total RNA and DNA were extracted and analyzed by HBV-specific RT-qPCR or qPCR. Results presented in graphs are the mean +/- SEM of 3 independent experiments ( 3 donors of $\mathrm{PHH}$ ) each performed in biological triplicate and are presented as ratio compared to the non-treated (NT) condition. $\mathrm{P} \leq$ $0.05^{*}, \mathrm{P} \leq 0.01^{* *}$, and $\mathrm{P} \leq 0.001^{* * *}$ were considered statistically significant.

Figure 3. LNP siPLK1 does not affect cccDNA amount. (A) A representative Southern blot, performed with pooled total DNA $(n=3)$ from Fig. $\mathbf{2}$ is shown Two micrograms of DNA were loaded per lane and separated into $1 \%$ agarose gel. Separated DNA were tranfered onto a nylon membrane and were hybridized with a digoxigenin labelled HBV probe, as described in detail in [22]. EcoRI digestion serves as control as it allows cccDNA linearization of HBV genotype D (shift from coiled $2.2 \mathrm{~kb}$ band to full lengh $3.2 \mathrm{~kb}$ linear DNA). (B) Quantification of Southern blot presented in panel B using image Lab software. Mitochondrial DNA was used as loading control and served for normalization. (C) Intracellular cccDNA specific Taqman qPCR [20] from PHH samples from Fig. 2, relative to non-treated (NT) condition. $\mathrm{P} \leq 0.05^{*}, \mathrm{P} \leq 0.01^{* *}$, and $\mathrm{P} \leq 0.001^{* * *}$ were considered statistically significant.

Figure 4. Pre-infection treatment with LNP siPLK1 in primary human hepatocytes. Experiments were conducted on three independent $\mathrm{PHH}$ batches. Drugs were administrated this time before and during the inoculation with HBV. In total 3 treatments were performed 3 days apart. The last treatment was comcomittant to the inoculation with HBV at 200vge/cell. LNP siPLK1 were used at indicated concentrations. LNP siHBV and LNP siCtrl were used at 200ng/mL and TFV at $10 \mu \mathrm{M}$. PreS1 peptide at 100nM served as an entry inhibitor. NT and NI are respectively infected/nontreated and non-infected/non-treated controls. Three days after infection (and 3 days after the last treatment), culture medium was changed (no treatement anymore) and cells were maintained 
for 4 additional days. (A) At day-7 post infection, RNA were extracted, reverse-transcribed and subjected to specific PLK1 qPCR. Normalization was done on PrP and quantification is relative to PLK1 expression in non-treated (NT) condition. (B) At the end of the experiment, secreted viral parameters were measured in the supernatants. RNA and DNA were extracted from viral particles and analyzed by HBV-specific RT-qPCR or qPCR, whereas levels of secreted ApoB, HBeAg and HBsAg were assessed by ELISA. (C) Intracellular total RNA and DNA extracted from HBV infected cells were analyzed by HBV-specific RT-qPCR or qPCR, normalized to housekeeping genes, and compared to non-treated (NT) condition, wheras intracellular cccDNA was analysed by specific Taqman qPCR [20]. Results presented in graphs are the mean of 3 independent experiments with SEM. $\mathrm{P} \leq 0.05^{*}, \mathrm{P} \leq 0.01^{* *}$, and $\mathrm{P} \leq 0.001^{* * *}$ were considered statistically significant.

Figure 5. Localization of HBV RNAs under LNP siPLK1 treatment. Differentiated HepaRG cells were infected with HBV at 200 vge/cell. After 7 days of infection cells were mock-treated (non treated control; NT) or treated 2 times 3 days apart with indicated molecules at indicated concentration. At day-13 post-infection (day-6 post treatment) total RNA were extracted, reverse transcribed and submitted to HBV specific qPCR. Cytosolic and nuclear compartments were recovered using a homemade protocol adapted from Weil et al., [21]. RNA were extracted using trizol and reverse transcribed. Home-designed primers were used to amplify the nuclear specific U6 snRNA and the Human Tyr-tRNA for the cytoplasm compartiment and served as purity control as well as housekeeping genes. Normalized on housekeeping gene and relative to non-treated (NT) condition. $\mathrm{P} \leq 0.05^{*}, \mathrm{P} \leq 0.01^{* *}$, and $\mathrm{P} \leq 0.001^{* * *}$ were considered statistically significant. 


\section{SUPPORTING FIGURE LEGENDS}

Supporting Figure 1. Efficacy of LNP siPLK1 and toxicity profile in HepG2 cells. (A) HepG2 cells were treated with increasing doses of LNP siPLK1, ranging from $1.3 \mathrm{ng} / \mathrm{mL}$ to $133 \mathrm{ng} / \mathrm{mL}$. RNA were extracted, reverse transcribed and PLK1 mRNA quantified using specific qPCR. Normalized to housekeeping gene and relative to Mock-Treated condition. (B) Cell viability was assessed with CellTiter Blue reagent and plotted among with knockdown of PLK1 RNA under various LNP siPLK1 concentrations.

Supporting Figure 2. Antiviral activity of LNP siPLK1 in non-transformed and non-dividing dHepaRG cells. Differntiated HepaRG were left uninfected (non-infected control; NI) or were infected with HBV genotype D at a multiplicity of infection of 200 vge/cell. From 7 days p.i., cells were mock-treated (non treated control; NT) or treated 2 times 3 days apart with indicated molecules. LNP siHBV and LNP siCtrl were used at $200 \mathrm{ng} / \mathrm{mL}$ and TFV at $10 \mu \mathrm{M}$. (A) RNAs were extracted and levels of PLK1 mRNAs (relative to PRNP mRNAs) analyzed by RT-qPCR. Results were normalized to the NT condition. (B) Three days after the last treatment (day-13 post-infection/day6 post-treatment), levels of secreted $\mathrm{HBeAg}$ and HBsAg were assessed by ELISA. (C) Intracellular total RNA and DNA were extracted and analyzed by HBV-specific RT-qPCR or qPCR. Results presented in graphs are the mean +/- SEM of 3 independent experiments ( 3 donors of $\mathrm{PHH}$ ) each performed in biological triplicate and are presented as ratio compared to the non-treated (NT) condition. $\mathrm{P} \leq 0.05^{*}, \mathrm{P} \leq 0.01^{* *}$, and $\mathrm{P} \leq 0.001^{* * *}$ were considered statistically significant.

Supporting Figure 3. Antiviral activity of LNP siPLK1. PHH from liver-humanized mice. Mice were perfused for 34 days to obtain $\mathrm{PHH}$ and were infected with $1.10^{9} \mathrm{HBV}$ genotype $\mathrm{D}$. Liver was digested by in situ perfusion and PHH seeded into 24-well plates. LNP treatments were conducted every 4 days starting at Day 0 and $\mathrm{BI}-2536$ treatment was started on Day 0 and replenished every 
tow days. Prior to treatments, the culture supernatants were collected and analysed for secreted HBV DNA and HBsAg levels. Secreted HBV DNA at D16 (Ai) and HBsAg at D16 (Aii).

Supporting Figure 4. pPLK1 is preferentially found in the nucleus of infected PHH. Western Blot of nuclear extracts shown in Figure 1D with above the quantification method used with Image Lab software. The pPLK1-S ${ }_{137}$ / Lamin B1 ratio allows correction of uneven overall protein quantity between each condition. Quantifications are presented relative to Mock-Treated condition, as indicated by the green R. The right panel shows western blot of corresponding cytosolic extracts, $30 \mu \mathrm{g}$ prot./lane. Lamin B1 and $\beta$-Tub served as purity control of cyto/nucleus fractionation. 
Figure 1

A

KO efficiency in PHH

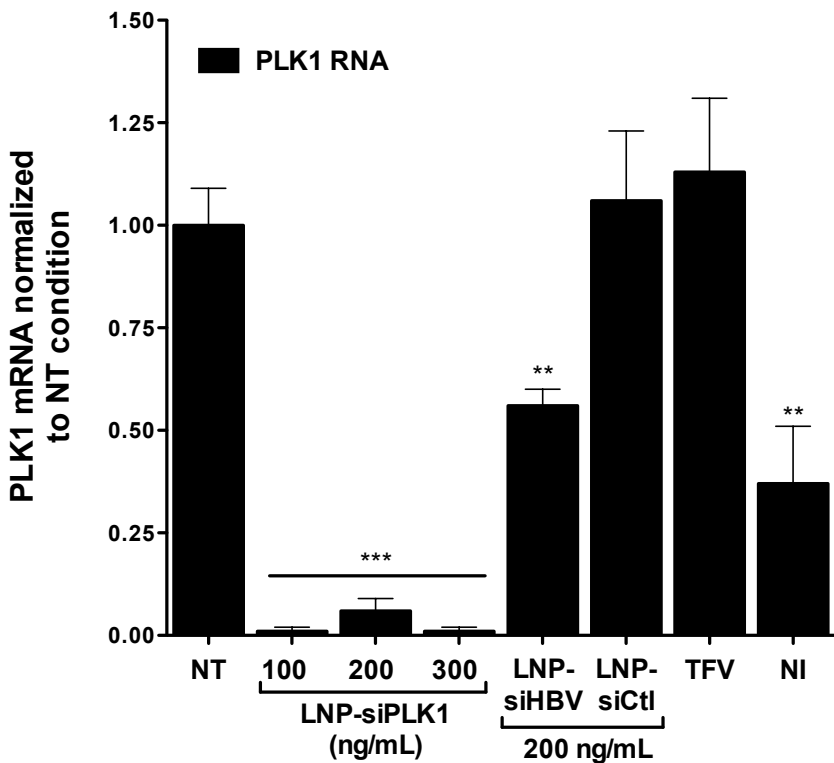

B

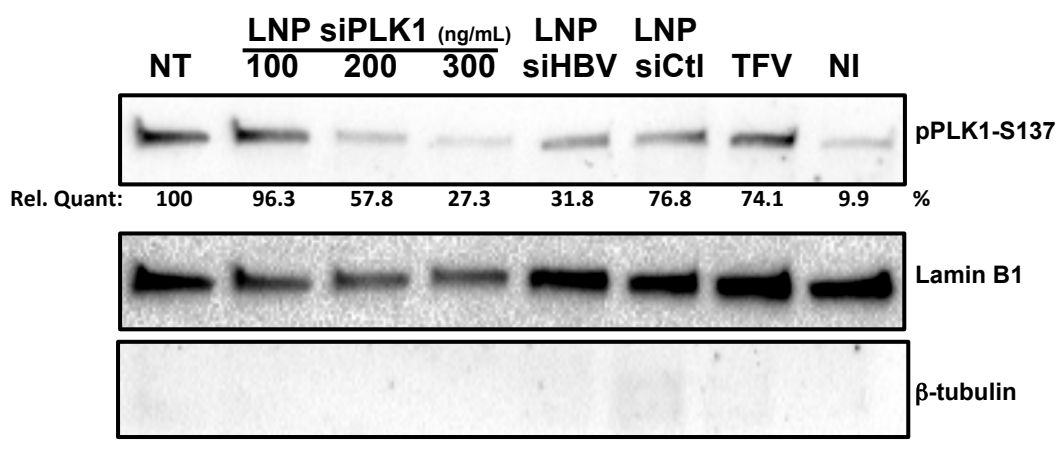

C Cytotoxicity

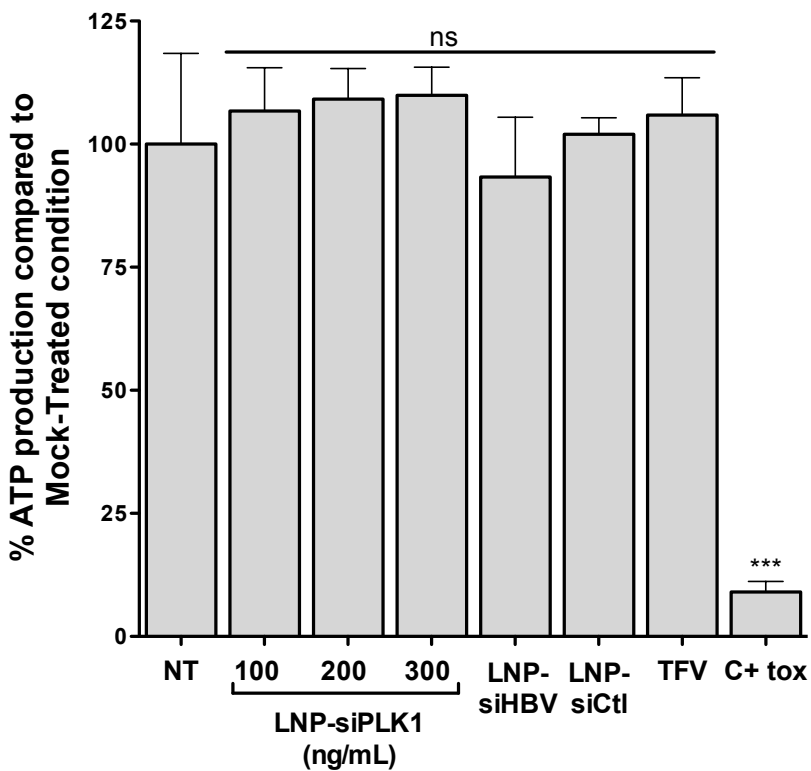




\section{Figure 2}

A

PHH- Txt post inf - HBV secreted parameters \& apoB

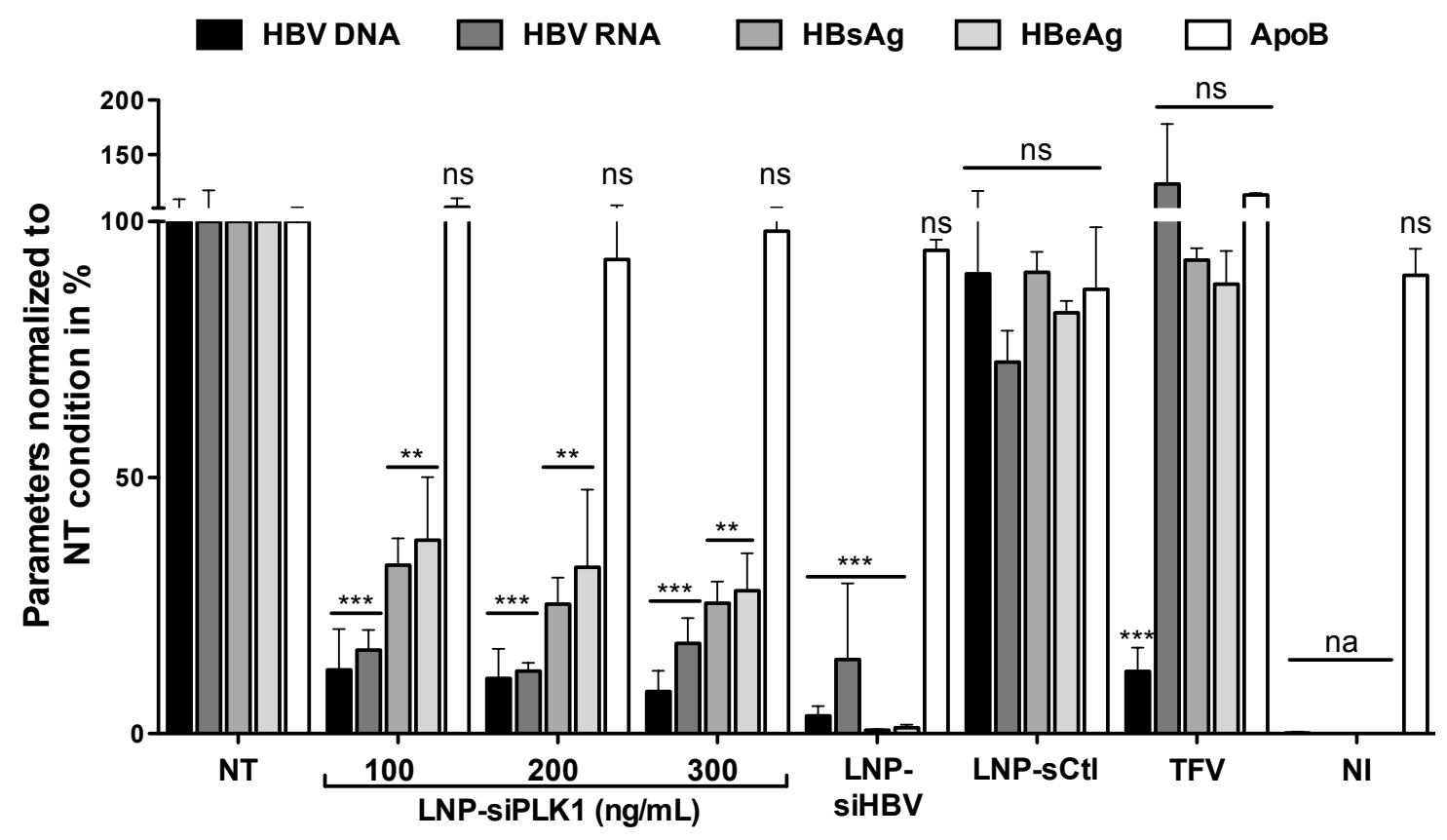

B

PHH - Txt post inf - HBV intracellular DNA and RNA

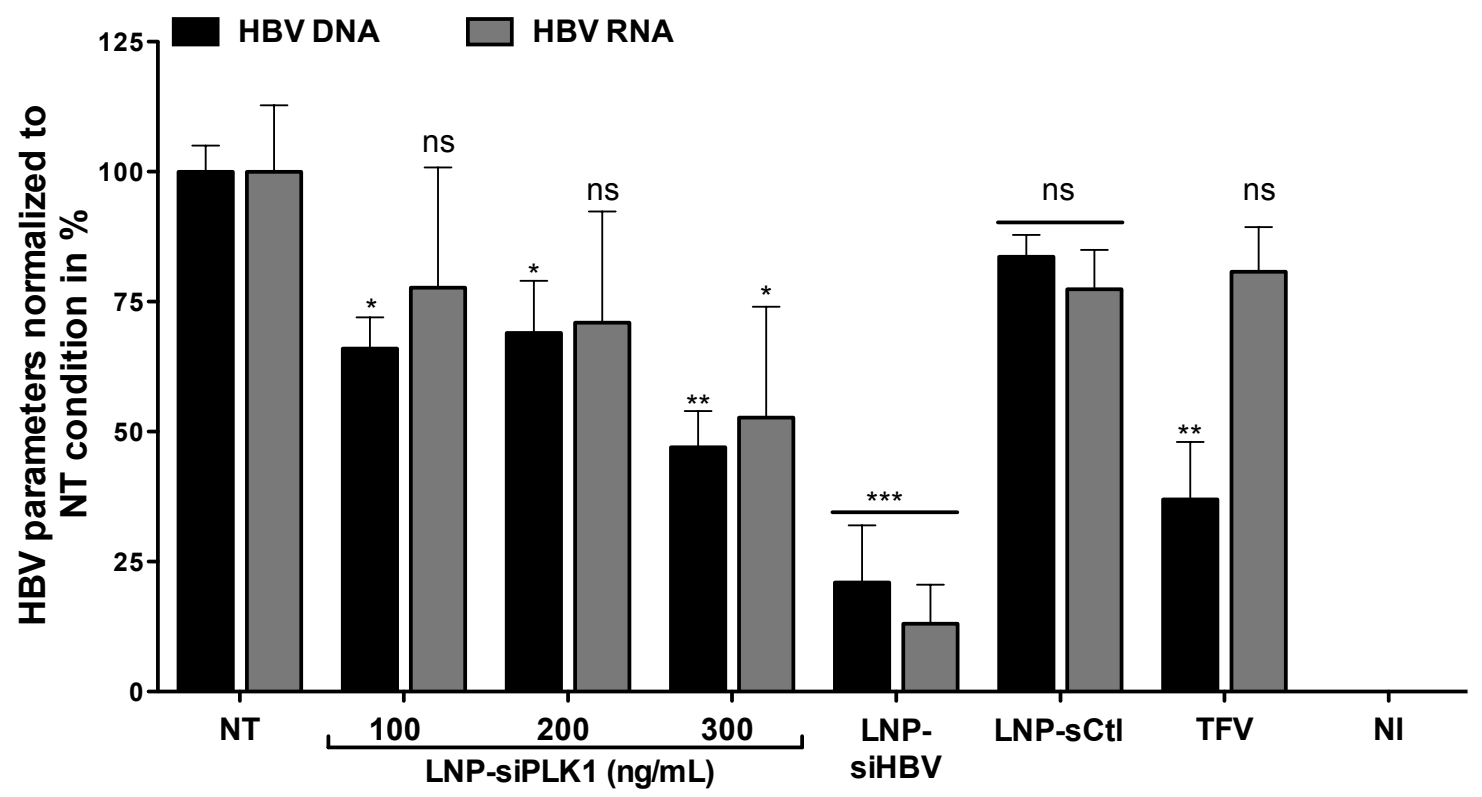




\section{Figure 3}

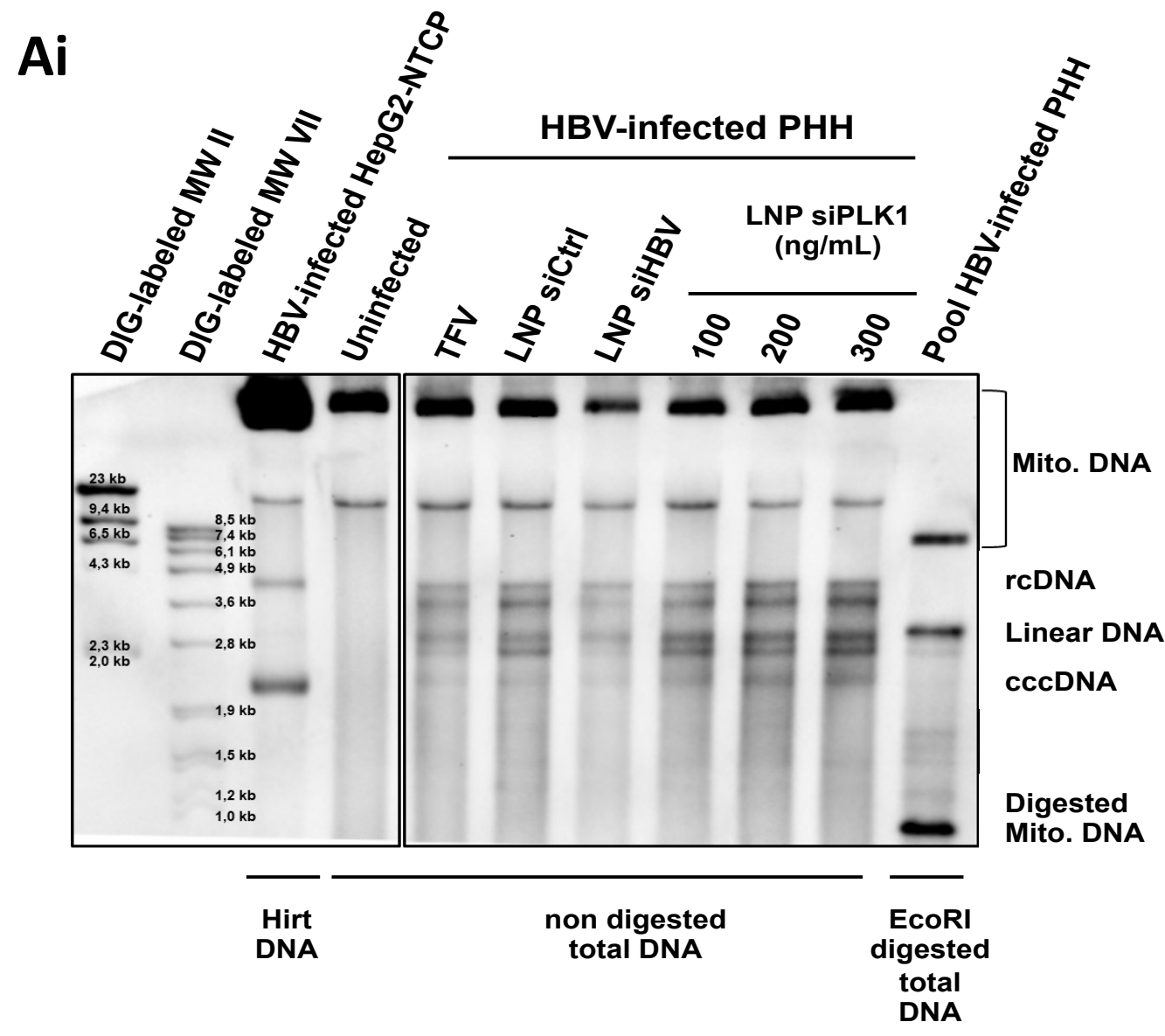

Aii

Quantif cccDNA from SB

B

Quantif cccDNA by qPCR
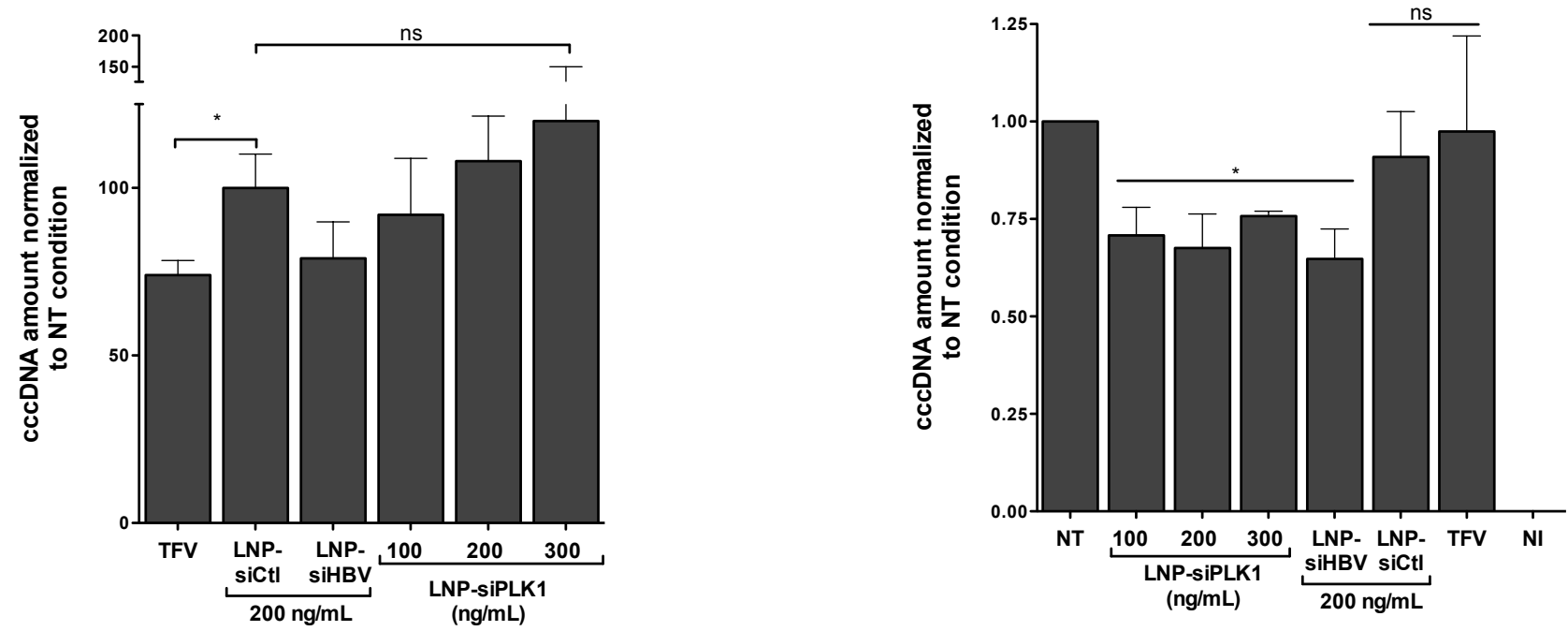
A

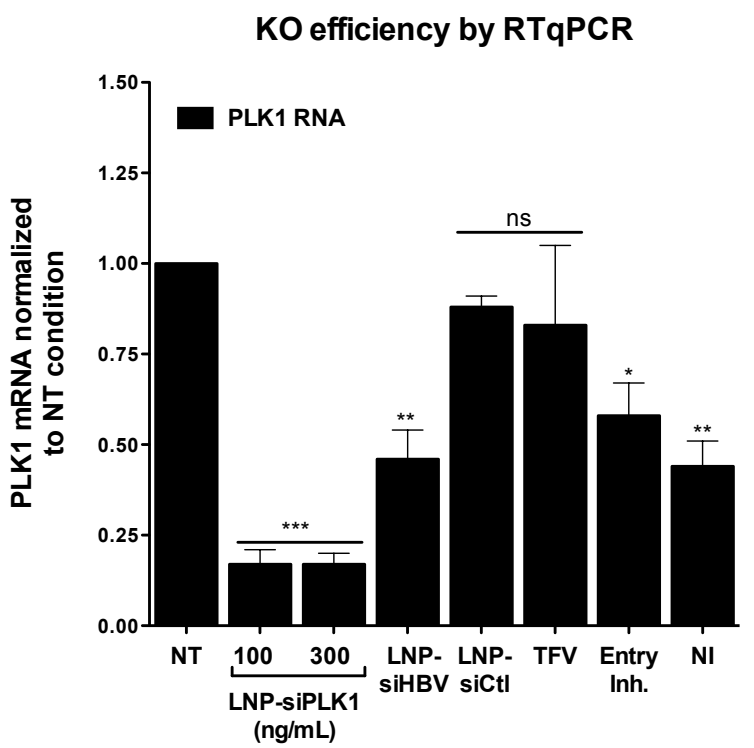

B

PHH - Txt pre inf - HBV secreted parameters

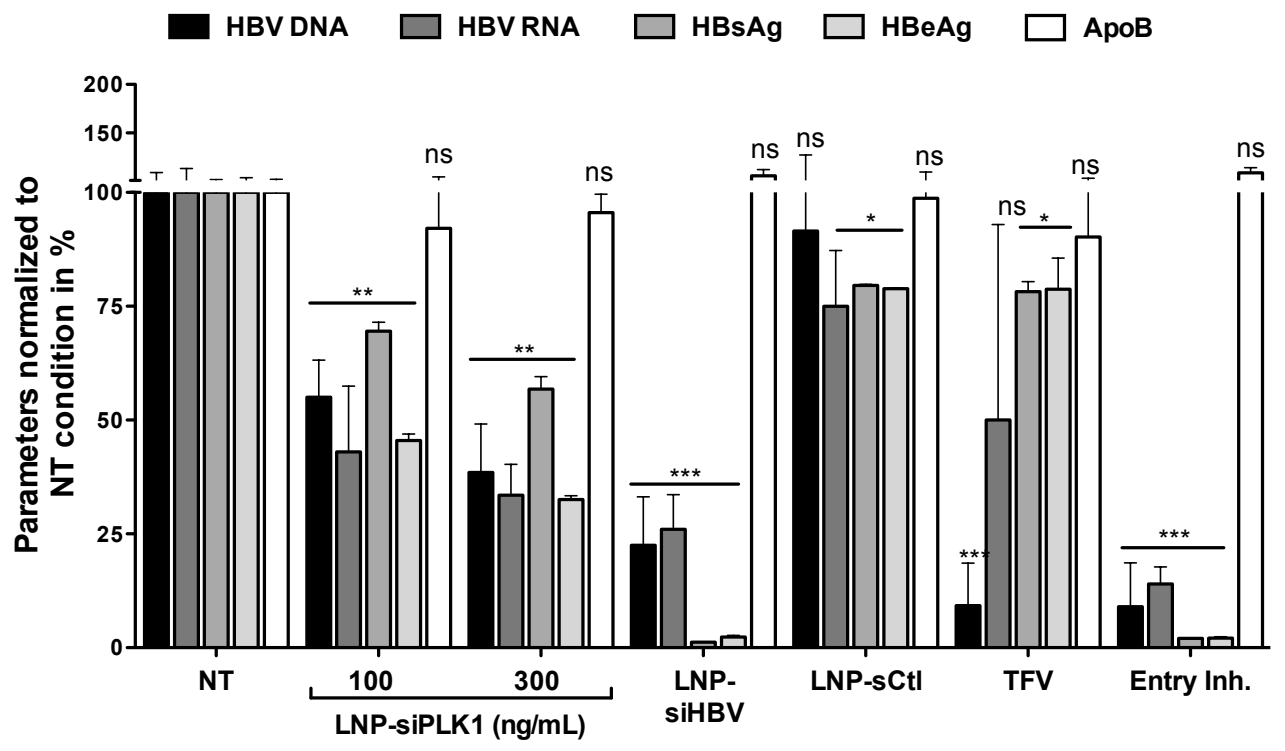

C

PHH - Txt pre inf - HBV intracellular parameters

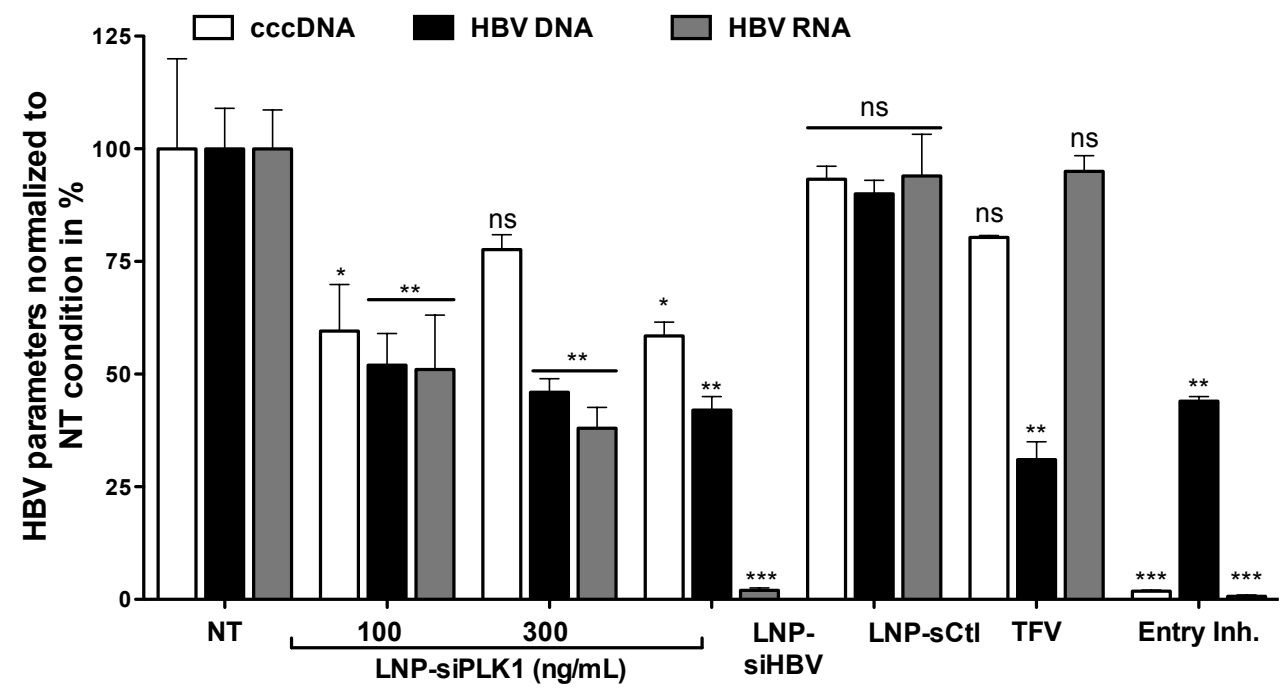


Figure 5

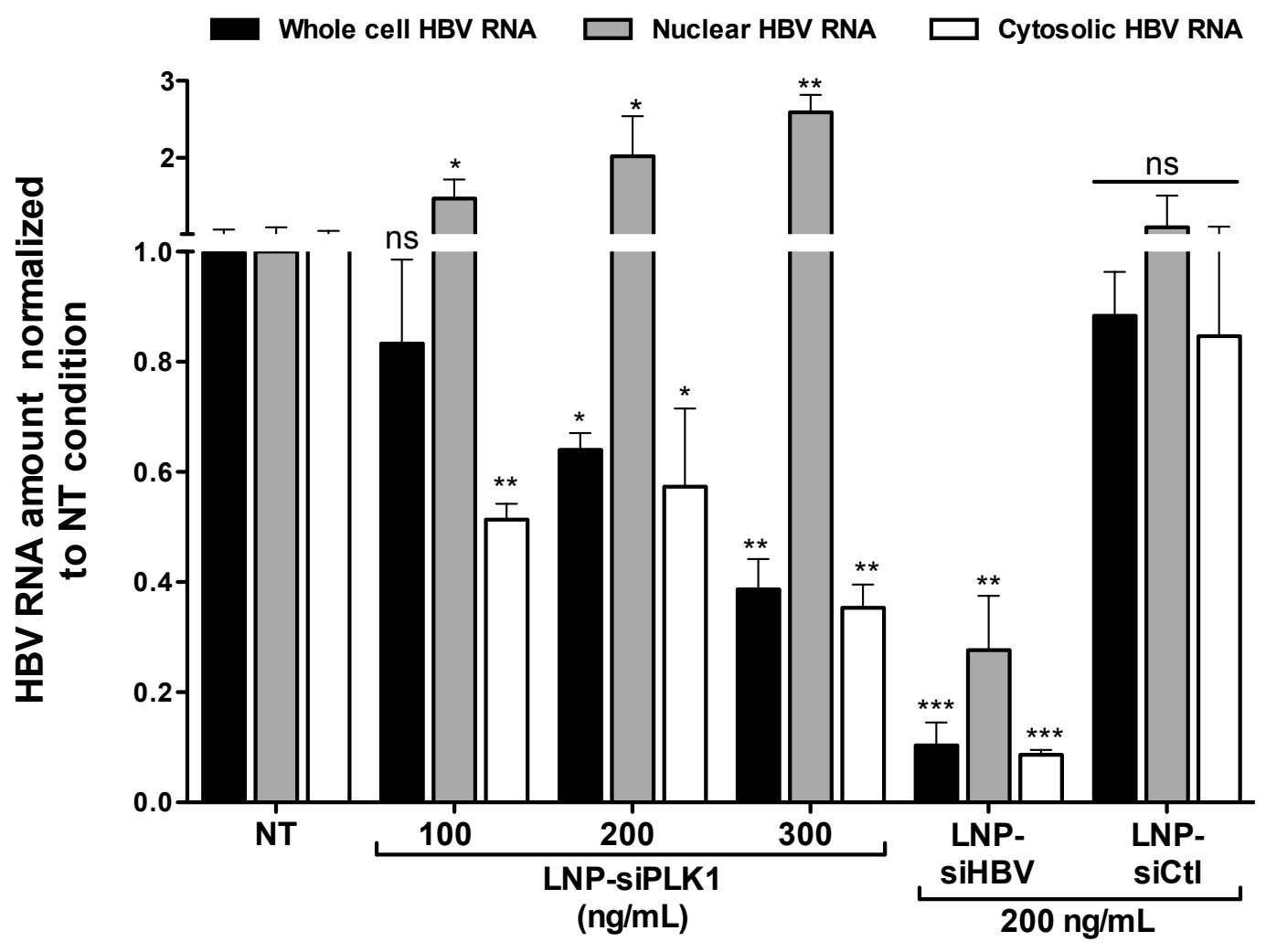


SUPPLEMENTAL FIGURES 


\section{Supplemental Figure 1}

A

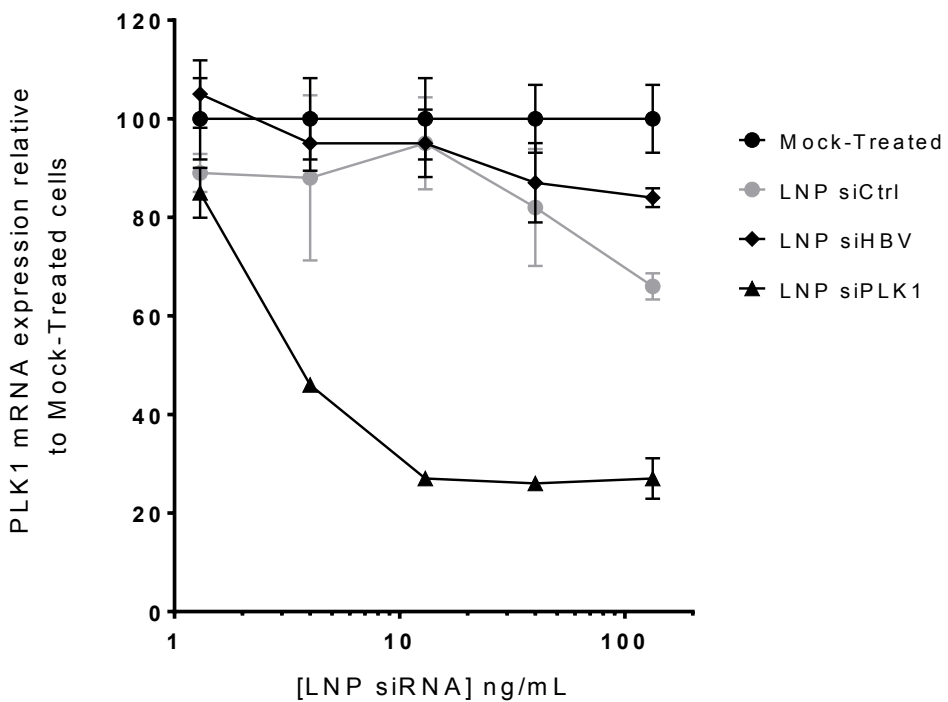

B

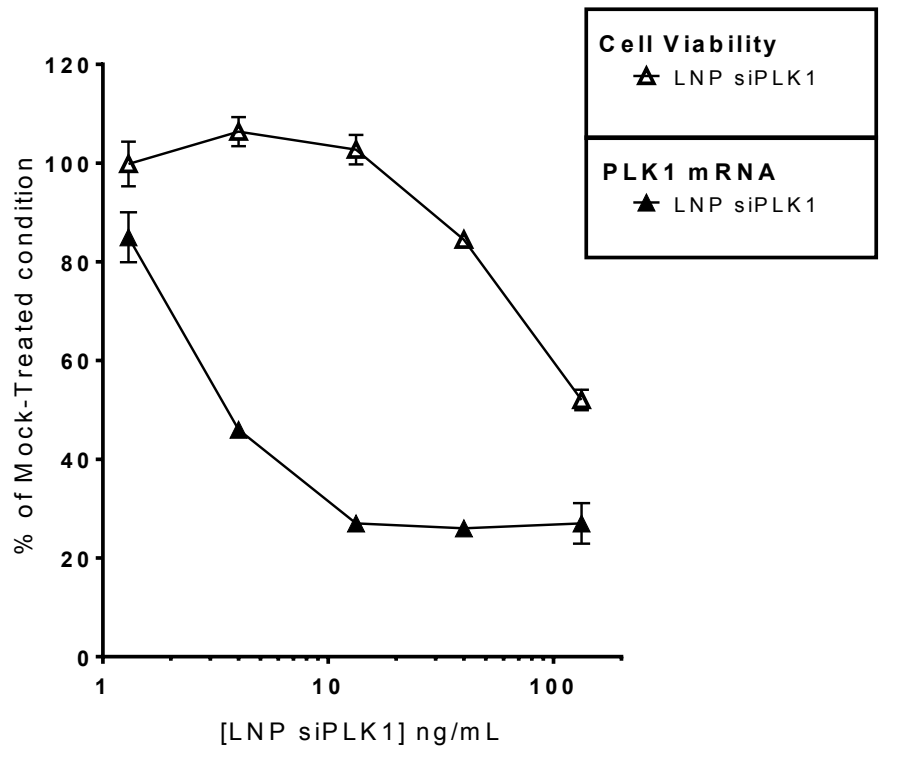




\section{Supplemental Figure 2}

A

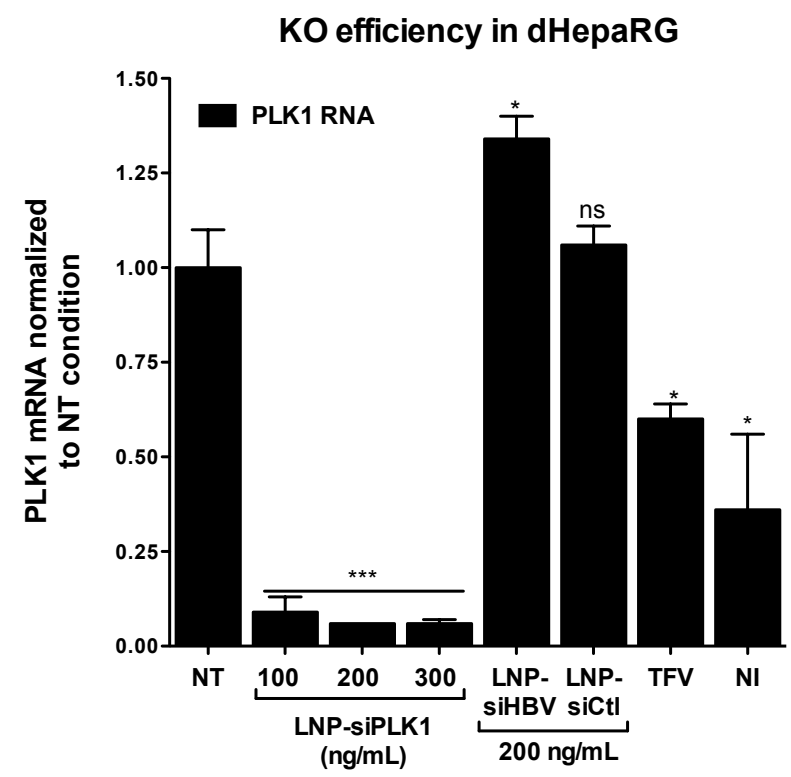

B

HepaRG - Txt post inf - HBV secreted parameters

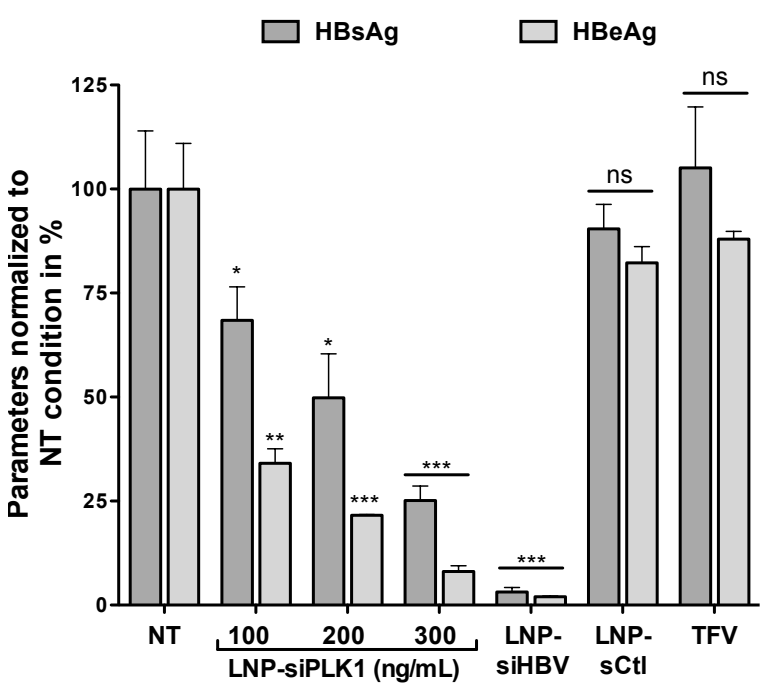

C

HepaRG - Txt post inf - HBV intracellular parameters

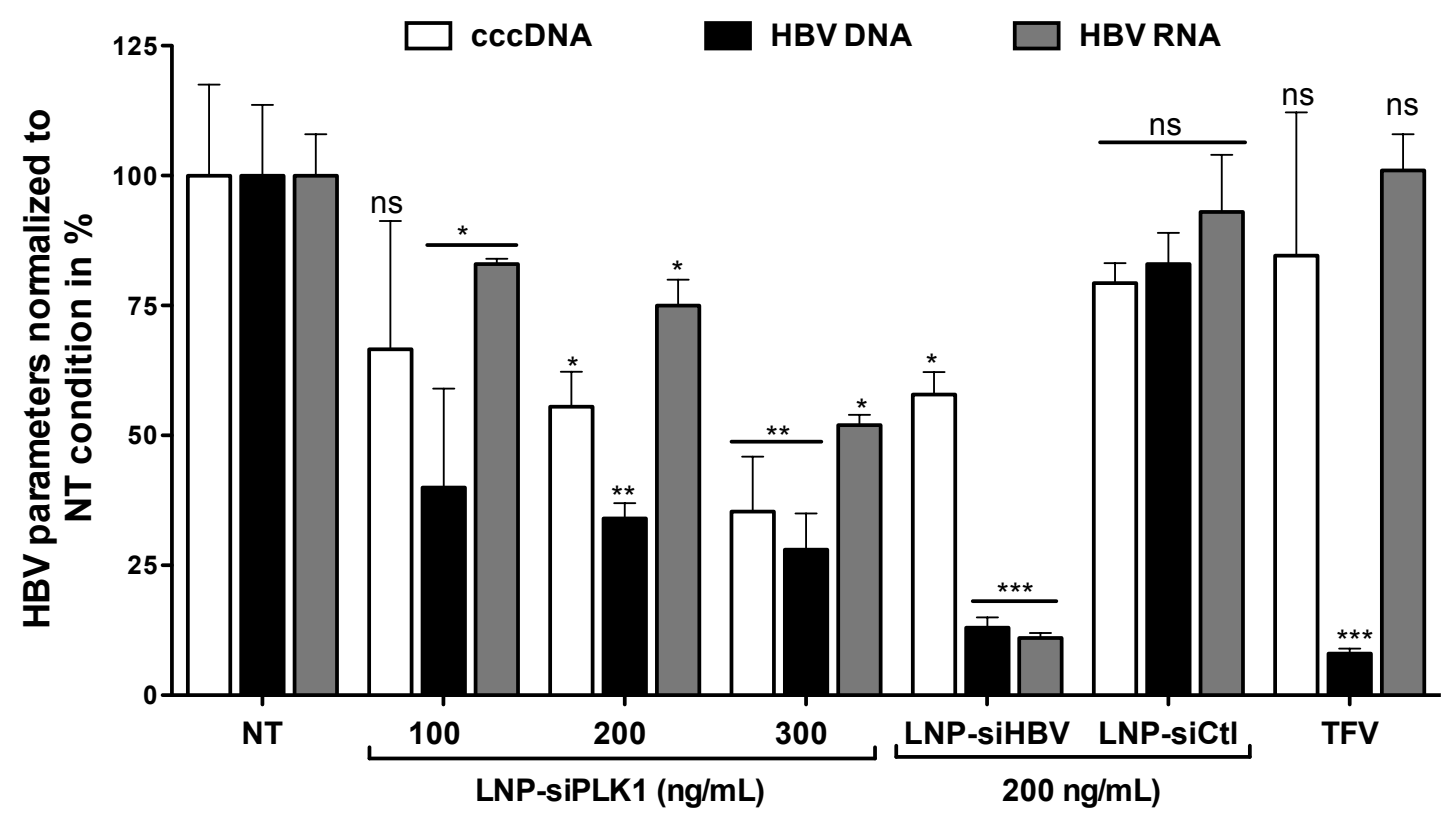




\section{Supplemental Figure 3}

Ai

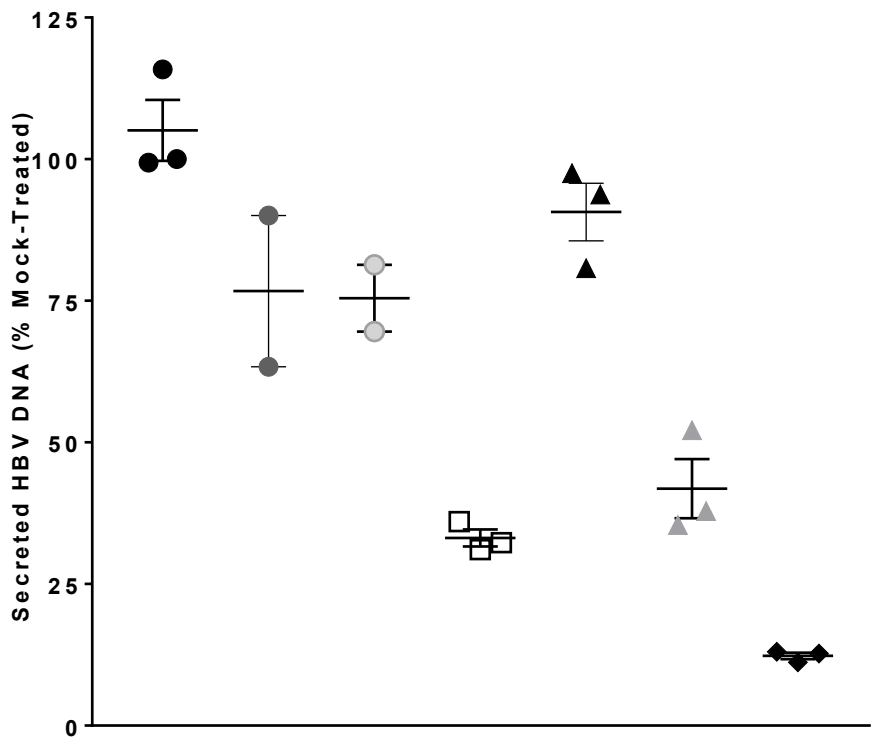

Mock-Treated

$\square \quad 500 \mathrm{nM} \mathrm{BI}-2536$

$\Delta \quad 100 \mathrm{ng} / \mathrm{mL}$ LNP siPLK 1

DMSO

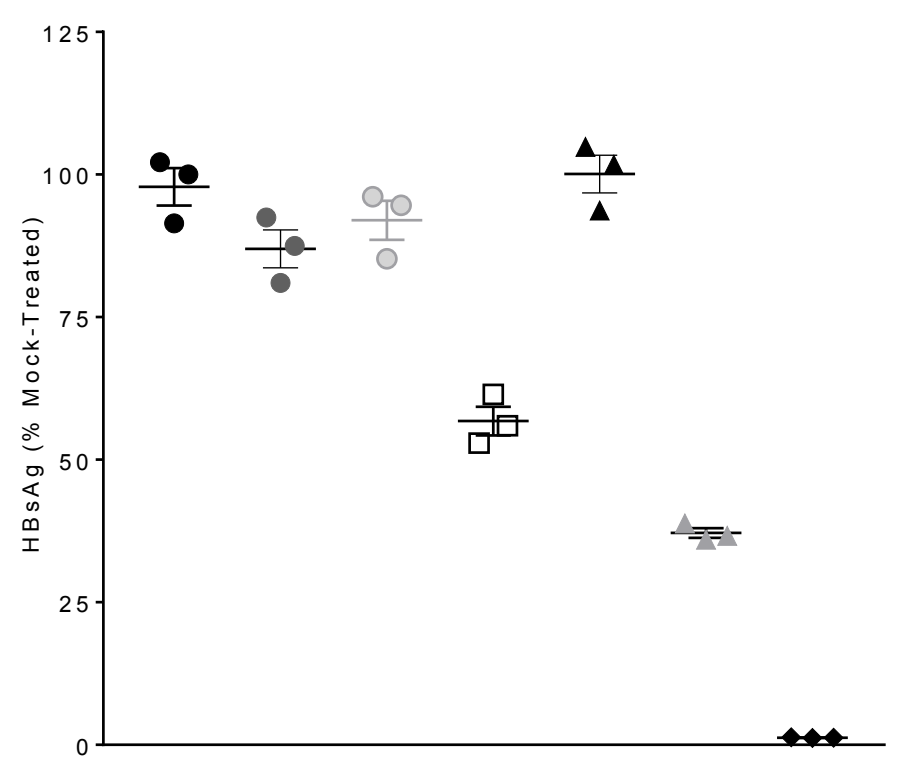

Aii
$100 \mathrm{ng} / \mathrm{mL}$ LNP siCtrl

A $300 \mathrm{ng} / \mathrm{mLLNP}$ siPLK 1

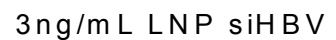




\section{Supplemental Figure 4}

\section{Nuclear extract}
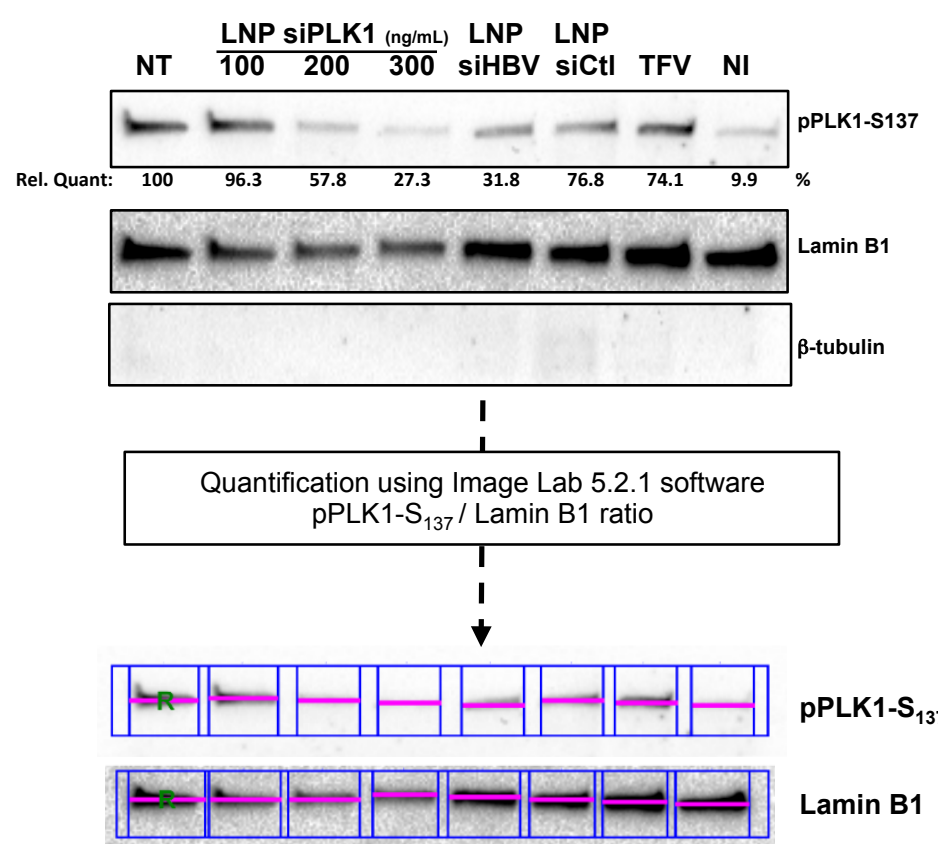

\section{Cytosolic extract}

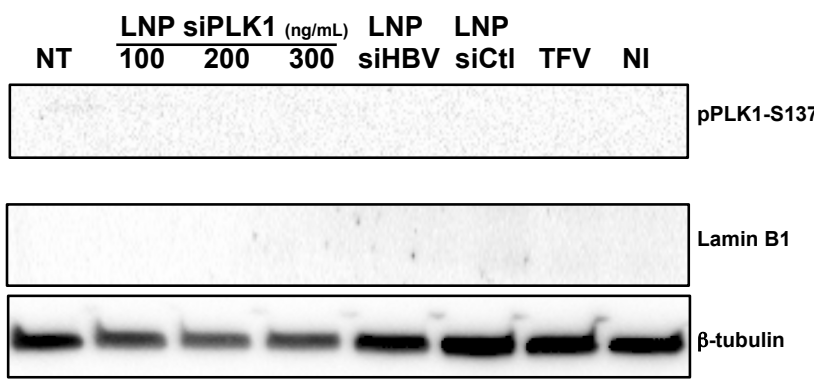

